UT-Komaba 11-9

October, 2011

\title{
On holographic three point functions for GKP strings from integrability
}

\author{
YOICHI KAZAMA ${ }^{\dagger}$ AND SHOTA KomATSU Ko $^{\ddagger}$ \\ Institute of Physics, University of Tokyo, \\ Komaba, Meguro-ku, Tokyo 153-8902 Japan
}

\begin{abstract}
Adapting the powerful integrability-based formalism invented previously for the calculation of gluon scattering amplitudes at strong coupling, we develop a method for computing the holographic three point functions for the large spin limit of Gubser-KlebanovPolyakov (GKP) strings. Although many of the ideas from the gluon scattering problem can be transplanted with minor modifications, the fact that the information of the external states is now encoded in the singularities at the vertex insertion points necessitates several new techniques. Notably, we develop a new generalized Riemann bilinear identity, which allows one to express the area integral in terms of appropriate contour integrals in the presence of such singularities. We also give some general discussions on how semiclassical vertex operators for heavy string states should be constructed systematically from the solutions of the Hamilton-Jacobi equation.
\end{abstract}

\footnotetext{
†kazama@hep1.c.u-tokyo.ac.jp

${ }^{\ddagger}$ skomatsu@hep1.c.u-tokyo.ac.jp
} 


\section{Contents}

1 Introduction $\quad 3$

2 Pohlmeyer reduction for strings in $\boldsymbol{A d S}_{3} \quad 8$

2.1 Basic formulation: A brief review . . . . . . . . . . . . . . . . 8

2.2 Case of GKP string in the large spin limit . . . . . . . . . . . . . . . . . . . . . 12

2.2.1 LSGKP solution in the embedding coordinate . . . . . . . . . . . . . . 13

3 Regularized area from integrability $r$

3.1 Definition of the regularized area . . . . . . . . . . . . . . . . . . 16

3.2 Comparison with the gluon scattering problem _ . . . . . . . . . . . . . . . 19

3.3 Generalization of Riemann bilinear identity . . . . . . . . . . . . . . . . . . . 21

3.3.1 From surface integrals to boundary contour integrals . . . . . . . . . . . . . 22

3.3.2 Double cover of the worldsheet for $\mathrm{N}$ point functions . . . . . . . . . . . . . . 23

3.3.3 Derivation of the generalized Riemann bilinear identity . . . . . . . . . . . 25

3.3.4 Simplification for the LSGKP string . . . . . . . . . . . . . . . . . . . . 29

3.4 Monodromy matrices and eigenfunctions for auxiliary linear problems . . . . . . . . . 30

3.4.1 Eigenvalues of monodromy matrices and "plus" and "minus" eigenvectors . . . . . 31

3.4.2 Calculation of explicit forms of monodromy matrices . . . . . . . . . . . . . . 32

3.4 .3 Eigenvectors of monodromy matrices . . . . . . . . . . . . . . . . 33

3.4.4 SL(2,C) invariant products between different eigenvectors . . . . . . . . . . . . 34

3.5 WKB analysis of the auxiliary linear problem . . . . . . . . . . . . . . . . 35

3.5.1 WKB expansions . . . . . . . . . . . . . . . . . 36

3.5.2 Small and big solutions and $\mathrm{SL}(2, \mathrm{C})$ invariant products of small solutions . . . . 38

3.6 Regularized area from SL(2,C)-invariant products . . . . . . . . . . . . . . . . . . . . . . 42

3.6.1 WKB curves for a specific parameter regime . . . . . . . . . . . . . . . 42

3.6.2 Relation between contour integrals and the products $\left\langle\boldsymbol{s}_{\boldsymbol{i}}, \boldsymbol{s}_{\boldsymbol{j}}\right\rangle \ldots \ldots$. . . . . 43

3.6.3 Connection between normalization and analyticity . . . . . . . . . . . . 45

3.6.4 Derivation of the formula for $\mathrm{SL}(2, \mathrm{C})$ products and computation of the regularized

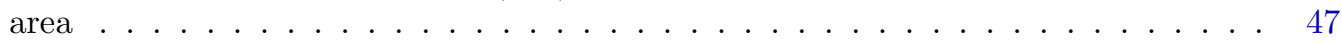

4 Semi-classical wave function and vertex operator for three-point functions 50

4.1 Structure of three-point functions . . . . . . . . . . . . . . . . . 50

4.2 Semi-classical vertex operator from WKB wave function . . . . . . . . . . . . . . 53

5 Summary and future directions $\quad 56$

$\begin{array}{ll}\text { Appendix A: Regularized area for general configuration of } \kappa_{i} \text { 's } & 57\end{array}$ 


\section{Introduction}

AdS/CFT [1-3] is perhaps the most profound and enigmatic paradigm of contemporary physics: Profound because it connects such fundamental yet disparate theories as gravity and non-gravitational gauge theories, defined in different dimensions and at diametric coupling strengths. It is enigmatic because even after more than a decade of intense research producing a heap of evidence, the gist of its mechanism has not been elucidated. Undoubtedly, deeper understanding of its dynamical aspect is needed in order to apply this powerful duality more reliably to interesting strong coupling phenomena in diverse areas of physics as well as to further unveil the rich intricate structure of string theory and M theory.

As in the prototypical example of this duality, the one between the $N=4$ super YangMills (SYM) theory in 4 dimensions and the type IIB superstring theory in $A d S_{5} \times S^{5}$ spacetime, a vast majority of the firm evidence for the AdS/CFT correspondence has been on the BPS quantities, which are protected from quantum effects by high degree of supersymmetry. Although research along such a direction will undoubtedly yield further interesting results, in order to reveal dynamical aspects of the duality it is important to employ methods which do not rely heavily on supersymmetry. Perturbative expansion is the simplest such method, which indeed has yielded many results on the gauge theory side (for a review [4]). On the string side, however, perturbative quantization is quite difficult, even for a single string, due to the curvature of the relevant spacetime. In any case, the inherent strong/weak nature of the duality calls for methods beyond perturbation theory.

A powerful and promising framework which in principle can overcome, under certain circumstances, the shortcomings and difficulties described above is the use of integrability and the associated analyticity. Of course fully integrable theories are scarce but since there can be various sensible perturbations of such theories they are not expected to be tied to exceptional dynamics.

Fortunately, both sides of the celebrated $\mathrm{SYM}_{\mathcal{N}=4} /$ AdS-string duality appear to possess integrable properties. Although the conjectured full integrability of the $\mathcal{N}=4 \mathrm{SYM}$ theory (see [5-7] and for a review [8] and references therein) is still to be confirmed and that of the quantum AdS string is yet to be formulated [10], at least the integrability of classical strings in $A d S_{5} \times S^{5}$ [9] (and in other similar backgrounds) is well-defined and firmly established.

In this article, we will describe an application of such an integrability to the computation of holographic three point functions of semi-classical string states, in particular the large spin limit of the Gubser-Klebanov-Polyakov(GKP) folded strings [11] rotating 
in $A d S_{3}$. Our study is strongly inspired by the work of Alday and Maldacena $[12,13]$ and the related works [14-16], which developed a beautiful method of computing the gluon scattering amplitudes at large 't Hooft coupling $\lambda$ by mapping it to a minimal surface problem for null polygonal Wilson loop in T-dualized $A d S_{5}$ and making clever use of the integrability of the classical string in $A d S$ space. The most ingenious feature of this method is that the information of the area can be extracted without the explicit knowledge of the form of the minimal surface. We will demonstrate that, with certain non-trivial modifications, to be explained shortly, the basic framework of this powerful method can be transplanted to the important problem of the evaluation of the aforementioned three point functions ${ }^{1}$.

Needless to say, understanding of the three point functions of the gauge invariant composite operators in SYM and of the corresponding dual string states which emanate from and land on the boundary of $A d S$ space is of utmost importance, as they are the basic building blocks encoding the dynamics of conformal theory. On the SYM side, the couplings of the BPS operators were computed already at the early stage [17], while those involving non-BPS operators have been studied more recently using weak coupling expansion and integrability structure [18-23]. On the string side, the result for the BPS operators was again obtained in [17] using type IIB supergravity, which agreed precisely with the SYM counterpart. As for non-BPS states, due to the lack of perturbative quantization scheme, even the computation of two point functions remained difficult. Rather recently, the situation was improved for heavy semi-classical states by the development of the saddle point method [24-26], which makes use of the known classical configurations. Subsequently, by inserting a light BPS operator (for which the back reaction can be neglected) into the heavy two point functions, various three point functions of heavy-heavy-light type have been computed [28-41]. Also, when a saddle point can be approximated by point like geodesics, such as in the case of BMN or near BMN strings, calculation of three point functions was performed in [42]. Treatment of the most interesting cases of three heavy operators has not been possible along the same line since no exact three-pronged solution in $A d S \times S$ spacetime is known to date.

This is precisely the situation where the powerful method developed for the gluon scattering problem may be utilized. As we already announced, we will deal with the case where each of the three external states corresponds to a GKP string with a large spin propagating within $A d S_{3}$. Since we will employ the saddle point approximation, the main problem is that of finding the minimal area surface in $A d S_{3}$ with prescribed behavior at the three punctures representing the external states. This is essentially the same

\footnotetext{
${ }^{1}$ Recently, a work in which three point functions of heavy strings in $A d S_{2} \times S^{k}$ were studied by similar means appeared [44]. We will make a brief comparison of this work with ours at the end of this section.
} 
type of problem encountered in the gluon scattering computation. The only difference is that in that case the information about the external gluons is mapped by T-duality to the behavior of the surface along the null polygonal boundaries at infinity, instead of at the punctures. Thus one expects that the same basic techniques, i.e. the Pohlmeyer reduction [43] and the analysis of the associated auxiliary linear problem with a spectral parameter $\xi$, are applicable.

To see more precisely how and to what extent such techniques can be applied, we must first describe the basic structure of the type of three point function of our interest in the saddle point approximation. Schematically, it can be written as

$$
G\left(x_{1}, x_{2}, x_{3}\right)=e^{-S\left[X_{*}\right]} \prod_{i=1}^{3} V_{i}\left[X_{*} ; z_{i}, x_{i}, Q_{i}\right] .
$$

Here, the arguments $x_{i}$ of the correlation function $G$ are the coordinates of the boundary of the (Euclideanized) $A d S_{3} . S\left[X_{*}\right]$ is the value of the action at the saddle point configuration $X_{*}$ and is of the order $\sqrt{\lambda}$, where $\lambda$ is the large 't Hooft coupling constant. $V_{i}$ denotes the vertex operator inserted at the point $z_{i}$ on the worldsheet ${ }^{2}$. It carries some large charge $Q_{i}$ so that, evaluated at the saddle configuration $X_{*}, \ln V_{i}$ is also of the order $\sqrt{\lambda}$.

In the case of the gluon scattering, due to the T-duality transformation, the effect of the vertex operators is included in the behavior of the area $S\left[X_{*}\right]$ at the null polygonal boundary at infinity so that one need not deal with $V_{i}$ part explicitly. The area $S\left[X_{*}\right]$ is infrared divergent and one can apply the integrability technique to evaluate the regularized area $S\left[X_{*}\right]_{r e g}$ after separating the divergence.

For a holographic three point function, $S\left[X_{*}\right]$ is also divergent, this time due to the presence of the pole singularities on the worldsheet at $z_{i}$. One can regularize and separate such a divergence and split the area as $\left.S\left[X_{*}\right]=S\left[X_{*}\right]_{\text {reg }}+S_{[} X_{*}\right]_{\epsilon}$, where $S\left[X_{*}\right]_{\text {reg }}$ is finite and $\epsilon$ stands for the regularization parameter. At the same time, the contribution of the vertex operator is also divergent for the same reason and it must be regularized in a similar way. This will be denoted by $V_{i}\left[X_{*}\right]_{\epsilon}$. In this way the correlation function above can be reorganized as

$$
G\left(x_{1}, x_{2}, x_{3}\right)=e^{-S\left[X_{*}\right]_{r e g}}\left[e^{-S\left[X_{*}\right]_{\epsilon}} \prod_{i=1}^{3} V_{i}\left[X_{*} ; x_{i}, Q_{i}\right]_{\epsilon}\right] .
$$

We will make an argument in Sec.4 that the contributions put together in the square brackets becomes finite and give an explicit example. There we also discuss the systematic procedure to construct the semi-classical vertex operator from the Hamilton-Jacobi

\footnotetext{
${ }^{2}$ The dependence on $z_{i}$ eventually disappears due to $S L(2, C)$ invariance.
} 
analysis. In the case of the GKP string, however, we have not yet been able to construct $V_{i}$ by such a procedure.

What we have been able to compute reliably by the integrability technique in this work is the "regularized area" $S\left[X_{*}\right]_{r e g}$. This computation, however, required certain non-trivial modifications of the method used in the gluon scattering.

One difference concerns the method of extraction of the correlation of the solutions in different regions of the worldsheet. For the present problem, different regions mean the vicinities of different singularities and the important information is obtained from the analysis of the local and the global monodromies around these singularities of a set of differential equations. In the gluon scattering case, the corresponding information is deduced from a system of functional equations (called T-system or Y-system) which relate the behavior of solutions in the so-called different Stokes sectors produced by the boundary conditions along the null polygon.

Another modification, which is more involved, is the necessity to derive a generalization of the Riemann bilinear identity, which is the key to express the area integral in terms of appropriate contour integrals on a double cover of the worldsheet. As we shall describe in detail in Sec. 3, the presence of double pole singularities will introduce additional logarithmic cuts in the relevant Riemann surface, a new feature not encountered in the gluon scattering problem. We have developed an appropriate generalization of the Riemann bilinear identity to systematically deal with this situation.

With these and other minor modifications, we can compute $S\left[X_{*}\right]_{\text {reg }}$ as a function of the parameters, which we call $\kappa_{i}$, in terms of which the AdS energy $E_{i}$ and the spin $S_{i}$ of the $i$-th GKP string are expressed.

The organization of the rest of this article is as follows. In section 2, we give a brief review of the formalism of the Pohlmeyer reduction and the associated auxiliary linear problem and describe the large spin limit of the GKP string solution in that framework. Section 3, which constitutes the main part of this paper, describes the method to compute the essential portion of the three point function for the GKP strings, namely the regularized area, defined in subsection 3.1. After making a comparison with the gluon scattering problem in subsection 3.2, we develop a generalized Riemann bilinear identity in subsection 3.3. We then analyze the monodromy matrices and the $S L(2, C)$-invariant products of the eigenfunctions for the auxiliary linear problem in subsection 3.4 and explicitly evaluate them in terms of the data extracted from the WKB solutions of the linear problem in subsection 3.5. Finally in subsection 3.6 we combine the result of subsequent subsections to obtain the formula for the regularized area in terms of the parameters which characterize the GKP strings. The structure of the remaining part of the three point 
function involving the vertex operators is discussed in section 4. Although the concrete result for the GKP strings is left for the future, we will describe the systematic procedure to construct the semi-classical vertex operators from the Hamilton-Jacobi analysis and give an instructive example. We conclude and indicate future directions in section 5 . An appendix is provided to give some technical details. This article became somewhat long as we tried to explain the intricate but essential analyses in as clear a fashion as possible. We hope that such scrupulous description will be beneficial to the reader.

Before ending the introduction, it is appropriate to make the following remark. As we were preparing the manuscript, the work of R. Janik and A. Wereszczyński [44] appeared, which computed the contribution of the AdS part to the correlation functions of three heavy strings in $A d S_{2} \times S^{k}$ by a similar method. Since the basic idea of adapting the powerful method developed for the gluon scattering problem is the same, our work has a sizable overlap with [44]. There are, however, a number of notable differences. (i) We deal with the GKP strings carrying large spin which propagate entirely in $A d S_{3}$ and hence with vanishing energy-momentum tensor. On the other hand, the work of [44] focuses on the $A d S_{2}$ part of the string carrying no spin but non-vanishing energy-momentum tensor that balances the contribution from the (unspecified motion in the) $S^{k}$ part. It is interesting to note, however, that, despite the difference of the systems considered, our result for the regularized area is very similar in structure to the $A d S$ part of the OPE coefficient obtained in [44], if the anomalous dimension $\Delta$ in [44] is identified with our $\kappa \propto E-S$, the difference of the $A d S$ energy and the spin of the GKP string ${ }^{3}$. (ii) In order to properly deal with the Riemann surfaces with logarithmic as well as square root cuts, we developed and systematically applied a new generalized Riemann bilinear identity. In contrast, the work of [44] did not need to consider logarithmic cuts. We believe that our method will be useful in further investigations, such as in the study of higher point functions. (iii) We make a strong use of the analyticity in the spectral parameter $\xi$ so that the $Z_{2}$ symmetry is not explicitly needed in the computations. (iv) We thoroughly investigate the cases with general configuration of parameters, which require non-trivial study of the behavior of the relevant WKB curves. These features make our work complementary to the preceding investigation [44].

\footnotetext{
${ }^{3}$ We thank R. Janik for pointing out this intriguing similarity.
} 


\section{Pohlmeyer reduction for strings in $\boldsymbol{A d S}_{3}$}

\subsection{Basic formulation: A brief review}

Just as in the computation of the gluon scattering amplitudes discussed in $[12,13]$, we will make full use of the framework of Pohlmeyer reduction to treat the strings in the $A d S_{3}$ space. So we begin by briefly reviewing this formalism. Except for a few places, we closely follow the notations of $[12,13]$.

A string in $A d S_{3}$ is described by the action on the Euclidean worldsheet, proportional to the area $A$, of the form

$$
S=\frac{T}{4} A=\frac{T}{2} \int d^{2} z\left(\partial X^{\mu} \bar{\partial} X_{\mu}+\Lambda\left(X^{\mu} X_{\mu}+1\right)\right)
$$

where $T$ is the string tension and $X_{\mu}=\vec{X}=\left(X_{-1}, X_{0}, X_{1}, X_{2}\right)$ are the embedding coordinates with the metric defined by $A^{\mu} B_{\mu}=\vec{A} \cdot \vec{B}=-A_{-1} B_{-1}-A_{0} B_{0}+A_{1} B_{1}+A_{2} B_{2}$. $\Lambda$ is the Lagrange multiplier enforcing the $A d S_{3}$ constraint $X^{\mu} X_{\mu}=-1$. The complex worldsheet (plane) coordinates are denoted by $z, \bar{z}$ and the derivatives are written as $\partial=\partial / \partial z, \bar{\partial}=\partial / \partial \bar{z}$. After eliminating $\Lambda$, the equation of motion for $X_{\mu}$ is given by

$$
\partial \bar{\partial} X_{\mu}=\left(\partial X^{\nu} \bar{\partial} X_{\nu}\right) X_{\mu}
$$

In addition we have the following Virasoro constraints:

$$
\partial X^{\mu} \partial X_{\mu}=\bar{\partial} X^{\mu} \bar{\partial} X_{\mu}=0
$$

The system is obviously invariant under the global isometry group $S O(2,2)$ acting on $X_{\mu}$ linearly.

The basic idea of the Pohlmeyer reduction is to describe the dynamics in terms of a convenient moving frame consisting of four basis vectors $\left\{q_{1}, q_{2}, q_{3}, q_{4}\right\}$ defined by

$$
\begin{aligned}
& q_{1}=\vec{X}, \quad q_{2}=e^{-\alpha} \bar{\partial} \vec{X}, \quad q_{3}=e^{-\alpha} \partial \vec{X}, \\
& q_{4}=\vec{N} \equiv \frac{1}{2} e^{-2 \alpha} \epsilon_{\mu \nu \rho \sigma} X^{\nu} \partial X^{\rho} \bar{\partial} X^{\sigma},
\end{aligned}
$$

where the $S O(2,2)$ invariant function $\alpha$ is defined by

$$
e^{2 \alpha} \equiv \frac{1}{2} \partial \vec{X} \cdot \bar{\partial} \vec{X}
$$

These basis vectors are normalized in the following way:

$$
q_{1}^{2}=-1, \quad q_{2} \cdot q_{3}=2, \quad q_{4}^{2}=1, \quad \text { other } q_{i} \cdot q_{j}=0
$$


Besides the function $\alpha$, the following two $S O(2,2)$ invariant quantities will be of fundamental importance ${ }^{4}$ :

$$
p \equiv+\frac{1}{2} \vec{N} \cdot \partial^{2} \vec{X}, \quad \bar{p} \equiv-\frac{1}{2} \vec{N} \cdot \bar{\partial}^{2} \vec{X}
$$

Now by using the equations of motion and the Virasoro conditions, one confirms that the $\partial$ and $\bar{\partial}$ derivatives of $q_{i}$ can all be expressed in terms of linear combinations of $q_{i}$ 's again. To express the result, it is convenient to introduce the $\mathrm{W}$ matrix consisting of $q_{i}$ in the following way:

$$
W_{\alpha \dot{\alpha}, a \dot{a}} \equiv \frac{1}{2}\left(\begin{array}{cc}
q_{1}+q_{4} & q_{3} \\
q_{2} & q_{1}-q_{4}
\end{array}\right)_{\alpha \dot{\alpha}} .
$$

Here $\alpha$ and $\dot{\alpha}$ denote the row and the column of the matrix, while the indices $a$ and $\dot{a}$ are associated with the bispinor representation of the each entry of $W$, which is a null $S O(2,2)$ vector. For example, an explicit representation for $q_{1}=\vec{X}$ can be taken to be

$$
\begin{aligned}
\left(q_{1}\right)_{a \dot{a}} & =X_{a \dot{a}}=X_{\mu} \sigma_{a \dot{a}}^{\mu}=\left(\begin{array}{cc}
X_{-1}+i X_{0} & X_{1}+i X_{2} \\
X_{1}-i X_{2} & X_{-1}-i X_{0}
\end{array}\right) \\
\sigma^{\mu} & =\left(1, i \sigma_{3}, \sigma_{1},-\sigma_{2}\right)
\end{aligned}
$$

Then the derivatives of $W$ can be related back to $W$ itself as

$$
\begin{aligned}
& \partial W+B_{z}^{L} W+W\left(B_{z}^{R}\right)^{T}=0, \\
& \bar{\partial} W+B_{\bar{z}}^{L} W+W\left(B_{\bar{z}}^{R}\right)^{T}=0
\end{aligned}
$$

which we call $W$-equations. The $2 \times 2$ matrices $B_{z}^{L}, B_{\bar{z}}^{L}, B_{z}^{R}, B_{\bar{z}}^{R}$ appearing here are given by

$$
\begin{aligned}
B_{z}^{L}=\left(\begin{array}{cc}
\frac{1}{2} \partial \alpha & -e^{\alpha} \\
-p e^{-\alpha} & -\frac{1}{2} \partial \alpha
\end{array}\right), & B_{\bar{z}}^{L}=\left(\begin{array}{cc}
-\frac{1}{2} \bar{\partial} \alpha & -\bar{p} e^{-\alpha} \\
-e^{\alpha} & \frac{1}{2} \bar{\partial} \alpha
\end{array}\right), \\
B_{z}^{R}=\left(\begin{array}{cc}
-\frac{1}{2} \partial \alpha & p e^{-\alpha} \\
-e^{\alpha} & \frac{1}{2} \partial \alpha
\end{array}\right), & B_{\bar{z}}^{R}=\left(\begin{array}{cc}
\frac{1}{2} \bar{\partial} \alpha & -e^{\alpha} \\
\bar{p} e^{-\alpha} & -\frac{1}{2} \bar{\partial} \alpha
\end{array}\right) .
\end{aligned}
$$

$B$ 's are traceless and hence are called $S L(2)$ connections. The $W$-equations contain the information of the equations of motion and the Virasoro conditions for the invariants $\alpha, p$ and $\bar{p}$. They read

$$
\begin{aligned}
& \partial \bar{\partial} \alpha-e^{2 \alpha}+p \bar{p} e^{-2 \alpha}=0 \\
& \partial \bar{p}=\bar{\partial} p=0
\end{aligned}
$$

\footnotetext{
${ }^{4}$ Because various equations in section 3 will become simpler, the signs of $p$ and $\bar{p}$ are taken to be opposite to those of $[12,13]$.
} 
The first line is identified with a modified sinh-Gordon equation and the second line dictates that $p$ and $\bar{p}$ are holomorphic and anti-holomorphic respectively. Furthermore from the $W$-equations one can deduce that the $S L(2)$ connections are flat, namely

$$
\begin{aligned}
{\left[\partial+B_{z}^{L}, \bar{\partial}+B_{\bar{z}}^{L}\right] } & =0, \\
{\left[\partial+B_{z}^{R}, \bar{\partial}+B_{\bar{z}}^{R}\right] } & =0 .
\end{aligned}
$$

These conditions reproduce the equations (2.16) and (2.17). What is of crucial importance is that one can generalize these flat connections to include a complex spectral parameter $\xi$ in the following way:

$$
\begin{array}{rlrl}
B_{z}(\xi) & =\frac{1}{\xi} \Phi_{z}+A_{z}, \quad B_{\bar{z}}(\xi)=\xi \Phi_{\bar{z}}+A_{\bar{z}} \\
A_{z} & \equiv\left(\begin{array}{cc}
\frac{1}{2} \partial \alpha & 0 \\
0 & -\frac{1}{2} \partial \alpha
\end{array}\right), & A_{\bar{z}} \equiv\left(\begin{array}{cc}
-\frac{1}{2} \bar{\partial} \alpha & 0 \\
0 & \frac{1}{2} \bar{\partial} \alpha
\end{array}\right), \\
0 & -e^{\alpha} \\
\Phi_{z} & \equiv\left(\begin{array}{cc}
0 & -\bar{p} e^{-\alpha} \\
-p e^{-\alpha} & 0
\end{array}\right), & \Phi_{\bar{z}} \equiv\left(\begin{array}{cc}
-e^{\alpha} & 0
\end{array}\right) .
\end{array}
$$

One can easily check that the flatness is preserved, namely

$$
\left[\partial+B_{z}(\xi), \bar{\partial}+B_{\bar{z}}(\xi)\right]=0
$$

This is the reflection of the fact that the system is integrable. These connections obey the following $Z_{2}$ symmetry relation:

$$
\sigma_{3} A \sigma_{3}=A, \quad \sigma_{3} \Phi \sigma_{3}=-\Phi
$$

As discussed in [13], this symmetry originates in the Virasoro constraints (2.3).

It is easy to see that these generalized connections are related to the original connections $B^{L}, B^{R}$ at special values of $\xi$. Explicitly,

$$
\begin{aligned}
B_{z}^{L} & =B_{z}(\xi=1), \quad B_{\bar{z}}^{L}=B_{\bar{z}}(\xi=1) \\
B_{z}^{R} & =\mathcal{U}^{\dagger} B_{z}(\xi=i) \mathcal{U}, \quad B_{\bar{z}}^{R}=\mathcal{U}^{\dagger} B_{\bar{z}}(\xi=i) \mathcal{U} \\
\mathcal{U} & =e^{i \pi / 4}\left(\begin{array}{ll}
0 & 1 \\
i & 0
\end{array}\right) .
\end{aligned}
$$

Now the flatness or the commutativity properties, (2.18) and (2.19), mean that the following "left" and "right" auxiliary linear problems can be consistently set up:

$$
\begin{array}{ll}
\partial \psi_{\alpha}^{L}+\left(B_{z}^{L}\right)_{\alpha}{ }^{\beta} \psi_{\beta}^{L}=0, & \bar{\partial} \psi_{\alpha}^{L}+\left(B_{\bar{z}}^{L}\right)_{\alpha}{ }^{\beta} \psi_{\beta}^{L}=0, \\
\partial \psi_{\dot{\alpha}}^{R}+\left(B_{z}^{R}\right)_{\dot{\alpha}}{ }^{\dot{\beta}} \psi_{\dot{\beta}}^{R}=0, & \bar{\partial} \psi_{\dot{\alpha}}^{R}+\left(B_{\bar{z}}^{R}\right)_{\dot{\alpha}}^{\dot{\beta}} \psi_{\dot{\beta}}^{R}=0 .
\end{array}
$$


There are two independent solutions for each of $\psi_{\alpha}^{L}$ and $\psi_{\dot{\alpha}}^{R}$, which will be denoted by $\psi_{\alpha, a}^{L}$ and $\psi_{\dot{\alpha}, \dot{a}}^{R}$, where $a=1,2, \dot{a}=\dot{1}, \dot{2}$. Then in matrix notation, the equations above read

$$
\begin{aligned}
\left(\partial+B_{z}^{L}\right) \psi_{a}^{L}=0, & & \left(\bar{\partial}+B_{\bar{z}}^{L}\right) \psi_{a}^{L}=0, \\
\left(\partial+B_{z}^{R}\right) \psi_{\dot{a}}^{R}=0, & & \left(\bar{\partial}+B_{\bar{z}}^{R}\right) \psi_{\dot{a}}^{R}=0 .
\end{aligned}
$$

If we use the $\xi$-dependent flat connections, one can consider instead

$$
\left(\partial+B_{z}(\xi)\right) \psi(\xi)=0, \quad\left(\partial+B_{\bar{z}}(\xi)\right) \psi(\xi)=0
$$

The $Z_{2}$ symmetry relation, (2.24), is reflected in the following property of $\psi(\xi)$.

$$
\left(\partial+B_{z}(\xi)\right) \sigma_{3} \psi\left(e^{i \pi} \xi\right)=0, \quad\left(\partial+B_{\bar{z}}(\xi)\right) \sigma_{3} \psi\left(e^{i \pi} \xi\right)=0
$$

Once we find $\psi(\xi)$, then $\psi^{L}$ and $\psi^{R}$ can be easily deduced from the relations between $B^{L}, B^{R}$ and $B(\xi)$ shown above, with the result

$$
\psi^{L}=\psi(\xi=1), \quad \psi^{R}=\mathcal{U}^{\dagger} \psi(\xi=i)
$$

The remaining important ingredient of the formalism is the reconstruction formula, which expresses the components of $W$, including in particular the original embedding coordinates $q_{1}=\vec{X}$, in terms of the solutions $\psi^{L}, \psi^{R}$ of the auxiliary linear problems. For this purpose, one needs to specify the normalization of $\psi^{L}$ and $\psi^{R}$. Define the $S L(2)$ invariant product for any pair of $S L(2)$ spinors $\psi$ and $\chi$ by

$$
\langle\psi, \chi\rangle \equiv \epsilon^{\alpha \beta} \psi_{\alpha} \chi_{\beta}, \quad\left(\epsilon^{\alpha \beta}=-\epsilon^{\beta \alpha}, \quad \epsilon^{12} \equiv 1\right) \text {. }
$$

Then, by using the equations of the auxiliary linear problems, (2.28) and (2.29), one can easily show that for the solutions of the auxiliary linear problems the products $\left\langle\psi_{a}^{L}, \psi_{b}^{L}\right\rangle$ and $\left\langle\psi_{a}^{R}, \psi_{b}^{R}\right\rangle$ are constant and anti-symmetric in $(a, b)$. Therefore one can normalize the solutions by the conditions

$$
\left\langle\psi_{a}^{L}, \psi_{b}^{L}\right\rangle=\epsilon_{a b}, \quad\left\langle\psi_{\dot{a}}^{R}, \psi_{\dot{b}}^{R}\right\rangle=\epsilon_{\dot{a} \dot{b}},
$$

where $\epsilon_{a b}$ is the anti-symmetric tensor with $\epsilon_{12} \equiv 1$.

With such normalized solutions, the entries of the $W$ matrix can be reconstructed succinctly as

$$
W_{\alpha \dot{\alpha}, a \dot{a}}=\psi_{\alpha, a}^{L} \psi_{\dot{\alpha}, \dot{a}}^{R}
$$

To prove it, first note that each entry of $W$ is a $S O(2,2)$ null vector, which has the bispinor representation in terms of the product of twistors as $p_{a \dot{a}}=\lambda_{a} \tilde{\lambda}_{\dot{a}}$. Therefore the 
components of $W$ can be written as $W_{\alpha \dot{\alpha}, a \dot{a}}=\lambda_{a, \alpha \dot{\alpha}} \tilde{\lambda}_{\dot{a}, \alpha \dot{\alpha}}$. Substituting this form into the $W$-equations, one finds that $\lambda_{a, \alpha \dot{\alpha}}$ and $\lambda_{\dot{a}, \alpha \dot{\alpha}}$ must be proportional to the solutions $\psi_{\alpha, a}^{L}$ and $\psi_{\dot{\alpha}, \dot{a}}^{R}$ of the linear problem ${ }^{5}$. Taking into account the normalization conditions (2.36) above, one obtains the formula (2.37).

The form of the formula (2.37) directly reflects the global symmetry of the target space. Clearly, the left and the right auxiliary linear problem separately admits linear transformation of the solutions. Taking into account the normalization conditions, this corresponds to the action of $S L(2, C)_{L} \times S L(2, C)_{R} \simeq S O(4, C)$ on the indices $a$ and $\dot{a}$ of the $W$ matrix. If we do note require the reality condition, this is precisely the action of the global symmetry on the embedding coordinates of the target space.

Before we finish this subsection, some remarks are in order.

- The method of the Pohlmeyer reduction does not by itself allow one to solve the non-linear problem. It merely transforms the equations of motion and the Virasoro constraints into reduced equations for smaller degrees of freedom, in this case the modified sinh-Gordon equation and the holomorphicity conditions for the invariant functions $\alpha, p$ and $\bar{p}$. If one can solve such reduced set of equations by other means, the solutions for the original variables can be readily reconstructed.

- However, as was demonstrated clearly in its application to the minimal surface problem relevant to gluon scattering, introduction of the complex spectral parameter $\xi$ into the auxiliary linear problem enormously strengthens the power of the formalism. The dependence on $\xi$, including the analyticity, encodes essential information so that if judiciously extracted one can compute important quantities without solving for the solutions explicitly. This feature is what we are going to utilize in the present problem, the computation of the three point function for the strings with large quantum numbers.

\subsection{Case of GKP string in the large spin limit}

Although the basic idea can be applied in principle to various semi-classical string states, in this work we will deal almost exclusively with the large spin limit of the GKP string propagating in $A d S_{3}$, which we shall call LSGKP. In this subsection, in order to make use of the integrability-based techniques in later sections, we will describe the LSGKP solution in the framework of Pohlmeyer reduction.

\footnotetext{
${ }^{5}$ Actually $\lambda_{a, \alpha \dot{\alpha}}$ does not depend on $\dot{\alpha}$ and $\tilde{\lambda}_{\dot{a}, \alpha \dot{\alpha}}$ does not depend on $\alpha$.
} 


\subsubsection{LSGKP solution in the embedding coordinate}

The LSGKP solution in the embedding coordinate is given using the Euclidean cylinder worldsheet variables $(\tau, \sigma)$ by

$$
\begin{gathered}
X_{-1} \pm i X_{0}=e^{ \pm \kappa \tau} \cosh \rho(\sigma), \\
X_{1} \pm i X_{2}=e^{\mp \kappa \tau} \sinh \rho(\sigma),
\end{gathered}
$$

where the function $\rho(\sigma)$, periodic with period $2 \pi$, has the profile depicted in the figure 2.1 . It is obtained from a smooth function expressible in terms of the Jacobi elliptic function

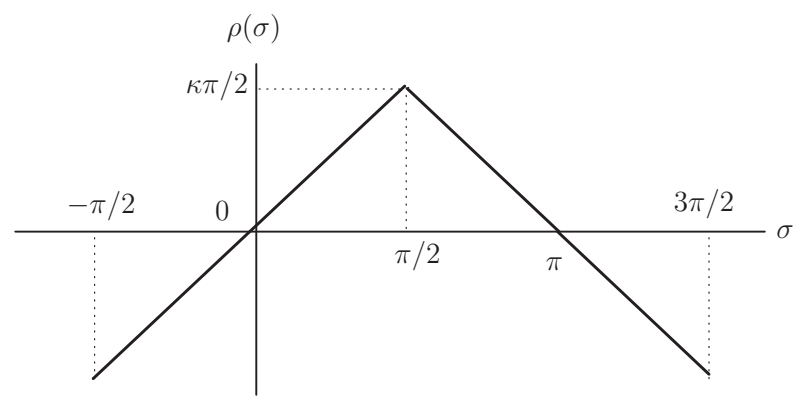

Figure 2.1: Profile of the function $\rho(\sigma)$ which appears in the large spin limit of the GKP solution.

by taking the limit of large $\kappa$. It is given in the interval $\left[-\frac{\pi}{2}, \frac{3 \pi}{2}\right]$ by

$$
\rho(\sigma)=\left\{\begin{array}{ll}
\kappa \sigma, & \left(-\frac{\pi}{2} \leq \sigma \leq \frac{\pi}{2}\right) \\
\kappa(\pi-\sigma), & \left(\frac{\pi}{2} \leq \sigma \leq \frac{3 \pi}{2}\right)
\end{array} .\right.
$$

In this limit we must treat the two segments separately and glue them together. There are two conserved quantities in this system, namely the AdS energy $E$ and the angular momentum (spin) $S$ in the 1-2 plane, which can be expressed in terms of the parameter $\kappa$ in the following way:

$$
\begin{aligned}
& E=T \kappa \int_{0}^{2 \pi} d \sigma \cosh ^{2} \rho=T(\kappa \pi+\sinh \kappa \pi), \\
& S=T \kappa \int_{0}^{2 \pi} d \sigma \sinh ^{2} \rho=T(-\kappa \pi+\sinh \kappa \pi) .
\end{aligned}
$$

Now, introducing the complex coordinates $w=\tau+i \sigma, \bar{w}=\tau-i \sigma$, it is straightforward to evaluate the basic invariant quantities $p(w), \bar{p}(\bar{w})$ and $\alpha(w, \bar{w})$ from their definitions (2.6) and (2.8) for this solution. The result is

$$
p(w)=-\frac{\kappa^{2}}{4}, \quad \bar{p}(\bar{w})=-\frac{\kappa^{2}}{4}, \quad e^{2 \alpha(w, \bar{w})}=\frac{\kappa^{2}}{4} .
$$


Going to the plane coordinate $z, \bar{z}$ defined by

$$
z=e^{w}, \quad \bar{z}=e^{\bar{w}}
$$

$p, \bar{p}$ and $e^{2 \alpha}$, which are $(2,0),(0,2)$ and $(1,1)$ forms respectively, become

$$
\begin{aligned}
p(z) & =-\frac{\kappa^{2}}{4 z^{2}}, \quad \bar{p}(\bar{z})=-\frac{\kappa^{2}}{4 \bar{z}^{2}}, \\
e^{2 \alpha(z, \bar{z})} & =\sqrt{p \bar{p}} .
\end{aligned}
$$

Recall that in the case of the gluon scattering problem, $p(z)$ is a polynomial in $z$ of degree $n-2$ for the scattering of $2 n$ gluons. Here it behaves as $\propto 1 / z^{2}$, with a double pole at the origin, expressing the emergence of a string at $z=0$ (or $\tau=-\infty$ ) from an appropriate vertex operator. Actually there is another pole at $z=\infty$, which can be seen by going to the inverse coordinate $z^{\prime}=1 / z$. In this coordinate $p\left(z^{\prime}\right)$ becomes $p\left(z^{\prime}\right)=-\kappa^{2} / 4 z^{\prime 2}$, showing the same structure at infinity. The relation (2.46) is characteristic of the large spin limit of the GKP solution and will play important roles.

We now study how the solutions of the linear auxiliary problem behave for LSGKP and check the reconstruction formula explicitly. These data will be important when we discuss the three point functions for LSGKP.

First it is convenient to simplify the linear problem by making the following gauge transformation:

$$
\psi=\mathcal{A} \tilde{\psi}, \quad \mathcal{A}=\left(\begin{array}{cc}
p^{-1 / 4} e^{\alpha / 2} & 0 \\
0 & p^{1 / 4} e^{-\alpha / 2}
\end{array}\right)
$$

Then, the equations $0=\left(\partial+B_{z}(\xi)\right) \psi,\left(\bar{\partial}+B_{\bar{z}}(\xi)\right) \psi=0$ drastically simplify in terms of the cylinder coordinates $w, \bar{w}$ :

$$
\begin{aligned}
& 0=\left[\partial_{w}-\frac{i \kappa}{2 \xi}\left(\begin{array}{ll}
0 & 1 \\
1 & 0
\end{array}\right)\right] \tilde{\psi}, \\
& 0=\left[\bar{\partial}_{w}+\frac{i \kappa \xi}{2}\left(\begin{array}{ll}
0 & 1 \\
1 & 0
\end{array}\right)\right] \tilde{\psi} .
\end{aligned}
$$

It is easy to solve these two equations, (2.48) and (2.49), and obtain two linearly independent solutions,

$$
\tilde{\psi}=\exp \left( \pm \frac{\kappa i}{2}\left(\xi^{-1} w-\xi \bar{w}\right)\right)\left(\begin{array}{c}
1 \\
\pm 1
\end{array}\right)
$$

Note that as we go around the origin once, this expression gets multiplied by a factor $e^{\mp \kappa \pi\left(\frac{1}{\xi}+\xi\right)}$. This is an important quantity characterizing the LSGKP string and will appear 
in the next section as the "local monodromy" datum. Applying the relation between $\psi(\xi=1, i)$ and $\psi^{L}, \psi^{R}$ given in (2.34), we obtain

$$
\begin{aligned}
\psi_{1}^{L}=\frac{1}{\sqrt{2}} e^{-\kappa i(w-\bar{w}) / 2}\left(\begin{array}{c}
-i e^{(w-\bar{w}) / 8} \\
e^{-(w-\bar{w}) / 8}
\end{array}\right), \quad \psi_{2}^{L}=\frac{1}{\sqrt{2}} e^{\kappa i(w-\bar{w}) / 2}\left(\begin{array}{c}
-e^{(w-\bar{w}) / 8} \\
i e^{-(w-\bar{w}) / 8}
\end{array}\right), \quad(2.51) \\
\psi_{\dot{i}}^{R}=-\frac{i e^{-i \pi / 4}}{\sqrt{2}} e^{\kappa(w+\bar{w}) / 2}\left(\begin{array}{c}
e^{-(w-\bar{w}) / 8} \\
e^{(w-\bar{w}) / 8}
\end{array}\right), \quad \psi_{\dot{2}}^{R}=-\frac{e^{-i \pi / 4}}{\sqrt{2}} e^{-\kappa(w+\bar{w}) / 2}\left(\begin{array}{c}
-e^{-(w-\bar{w}) / 8} \\
e^{(w-\bar{w}) / 8}
\end{array}\right) .
\end{aligned}
$$

It is easy to check that these solutions are properly normalized, namely $\left\langle\psi_{1}^{L}, \psi_{2}^{L}\right\rangle=$ $\left\langle\psi_{\dot{1}}^{R}, \psi_{\dot{2}}^{R}\right\rangle=1$.

With the solutions of the auxiliary linear problem at hand, we can now reconstruct the embedding coordinates of the LSGKP solutions. From the structure of $W$ matrix and the reconstruction formula $(2.37),\left(q_{1}\right)_{a \dot{a}}$ is given by

$$
\left(q_{1}\right)_{a \dot{a}}=\psi_{1 a}^{L} \psi_{\dot{1} \dot{a}}^{R}+\psi_{2 a}^{L} \psi_{\dot{2} \dot{a}}^{R}
$$

Then we obtain the simple result

$$
q_{1}=-\frac{1}{\sqrt{2}}\left(\begin{array}{cc}
e^{\kappa \tau} e^{\kappa \sigma} & e^{-\kappa \tau} e^{\kappa \sigma} \\
-e^{\kappa \tau} e^{-\kappa \sigma} & e^{-\kappa \tau} e^{-\kappa \sigma}
\end{array}\right)
$$

By making an appropriate $S L(2, C)_{L} \times S L(2, C)_{R}$ global transformation, this can be put into the familiar form (2.10), with $X_{-1} \pm i X_{0}$ and $X_{1} \pm i X_{2}$ given precisely by the standard LSGKP solution (2.38) and (2.39). Before closing this section, we would like to make some important remarks:

- If we regard $\tau$ as being real, the embedding coordinates of the solution described by (2.38) and (2.39) are not all real. The appearance of complex trajectories of this kind is often encountered in the saddle point calculation of correlation functions. In some cases, Wick rotation of the target space "time" coordinate, $X_{0} \rightarrow i X_{0, e}$, can be performed so that such solutions can be regarded as real solutions in the Euclidean $A d S$. However, this prescription does not apply universally. In fact in the present case, $X_{2}$ in (2.38) and (2.39) is also complex-valued and it is unnatural to make it real by another "Wick rotation". The viewpoint we adopt in this work is that the solution described by (2.38) and (2.39) is simply a complex saddle point of the path integral of the string theory in Lorentzian $A d S_{5}$. This should be the correct interpretation since what $A d S / C F T$ correspondence predicts is a duality between $\mathcal{N}=4$ super Yang-Mills theory in the Lorentzian signature and the superstring theory in the Lorentzian $A d S_{5} \times S^{5}$. 
- The embedding coordinates $\left(X_{-1}, X_{0}, X_{1}, X_{2}, X_{3}, X_{4}\right)$ of the $A d S_{5}$ space are related to the Poincaré coordinates $\left(x^{0}, x^{1}, x^{2}, x^{3}, z\right)$ as

$$
X_{-1}+X_{4}=\frac{1}{z}, \quad X_{-1}-X_{4}=z+\frac{x^{\mu} x_{\mu}}{z}, \quad X_{\mu}=\frac{x_{\mu}}{z} .
$$

Since we are considering the $A d S_{3}$ subspace spanned by $\left(X_{-1}, X_{0}, X_{1}, X_{2}\right)$, the coordinates $X_{3}$ and $X_{4}$ in (2.55) vanish in our case. This imposes a constraint of the form $x^{0} x_{0}+x^{1} x_{1}+x^{2} x_{2}+z^{2}=1$ on the Poincaré coordinates and hence the positions of the vertex operators on the boundary at $z=0$ are restricted to be in the region $x^{0} x_{0}+x^{1} x_{1}+x^{2} x_{2}=1$. If one wishes to avoid this constraint, one can instead consider solutions in $\left(X_{-1}, X_{1}, X_{2}, X_{4}\right)$ from the beginning by replacing $i X_{0}$ in (2.38) and (2.39) with $X_{4}$. With this choice of embedding coordinates, the equations (2.38) and (2.39) describe a folded spinning string which is emitted from the boundary of $A d S$ and gets absorbed at the horizon. We can then perform an appropariate $S L(2, C)_{L} \times S L(2, C)_{R}$ global transformation to make it into an appropriate saddle point configuration for a two point function on the boundary.

\section{Regularized area from integrability}

In this section, we shall describe in detail how one can compute one of the two building blocks of the semi-classical three point function, namely the contribution of the form $\exp \left(-S\left[X_{*}\right]\right)$, where $S\left[X_{*}\right]$ is the action (or the area) evaluated at the saddle point solution $X_{*}$ in the presence of vertex operators. As briefly mentioned in the introduction, $S\left[X_{*}\right]$ itself contains divergences coming from the vicinity of the points where the vertex operators are inserted. We shall discuss later in Sec. 4 that such divergences will in the end be canceled by the contribution of the vertex operators (or the wave functions), which is also divergent, and the correlation function as a whole will be finite. In what follows, we shall first clarify how we should compute the action in the case of LSGKP folded strings and then discuss the isolation of the divergent part of the area by using the knowledge of the behavior of the GKP strings near the insertion points. Subsequently, the remaining finite part of the area will be calculated by using a generalization of the Riemann bilinear identity and the WKB analysis of the auxiliary linear problems.

\subsection{Definition of the regularized area}

We begin by clarifying precisely what area we need to compute and how we will actually evaluate it. 
As was reviewed in the previous subsection, in the large spin limit, the GKP string propagates in completely folded fashion. As long as we stick to this limit this feature should continue to hold for the 3-pronged saddle configuration which is relevant for the three point function. Therefore the area swept out by one half of the folded string is the same as that swept out by the other half and we only need to compute one of them and multiply by a factor of two. This is depicted in figure 3.1. The worldsheet for such "half string" is like that of an open string and can be represented by the upper half plane, as shown in figure 3.1. Now a very convenient way to compute the area for this portion of the worldsheet is to first extend it smoothly to the whole complex plane, compute the area and then divide the result by two. But as we have to double this result at the end, the net result we want coincides with the contribution from the whole plane itself.

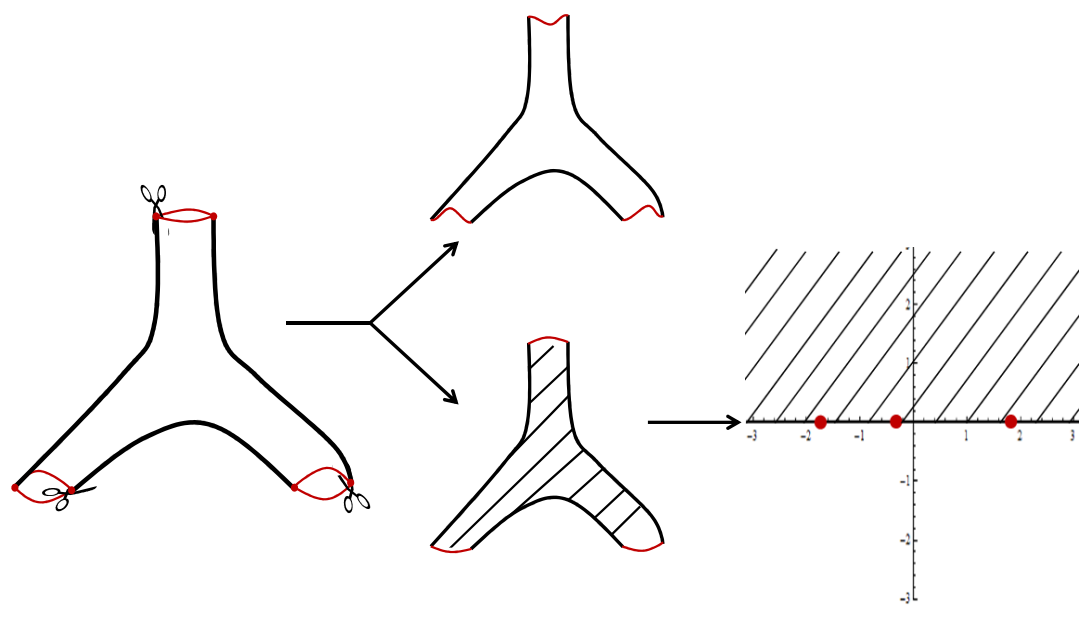

Figure 3.1: Splitting the worldsheet into two identical halves. Each sheet can be mapped to the upper half plane.

To justify this method, we should clarify a possibly confusing point. An important point is that the method above is valid in so far as we use it for the computation of the area, which is not affected by the non-trivial behavior of the actual string solution in the vicinity of the folding points. If one wishes to reconstruct the entire LSGKP solution including the acute folding behavior automatically using the integrability-based method of the previous section, one must modify the behavior of $\alpha(z, \bar{z})$ at the folding points and this in turn changes the monodromy of the solutions of the auxiliary linear problem, as exemplified in the work [27]. Therefore, if we are interested in the saddle point configuration itself for the three point function, we must either start from the exact form of the GKP string [11] and properly take into account the subtle behavior of $\alpha(z, \bar{z})$ etc. in the large spin limit, or find the solution for the half string from the analysis in the upper half plane and then paste its copy on top of it to construct the closed string 
solution. In either way we cannot use the smooth extension into the lower half plane as in the above method.

For the computation of the area, we can circumvent such complicated procedures by the simpler method described above. As is clear from figure 3.1, after the smooth extension, the worldsheet is symmetric under the reflection about the real axis and hence the solution $\alpha(z, \bar{z})$ of the modified sinh-Gordon equation must enjoy the same symmetry. Since the area is expressed in terms of $\alpha(z, \bar{z})$, the contribution from the lower half plane must equal that from the upper half plane. Moreover, since the area is conformally invariant, we can make an arbitrary conformal transformation to move the positions of the singularities (i.e. the positions of the vertex operators) from along the real axis to wherever are more convenient on the complex plane. We will make use of such a freedom in developing the method of computation later. The conclusion is that as far as the computation of the area is concerned we may effectively forget about the effect of folding.

The area can be expressed as

$$
A=2 \int d^{2} z \partial \vec{X} \cdot \bar{\partial} \vec{X}=4 \int d^{2} z e^{2 \alpha}
$$

As discussed in the previous section, near the insertion points $z_{i}$ the quantity $e^{2 \alpha}$ behaves like

$$
e^{2 \alpha} \simeq \sqrt{p \bar{p}}
$$

which in the case of GKP string goes like $1 /\left|z-z_{i}\right|^{2}$, giving rise to log divergences for the area. Thus one can separate the finite and the divergent part of the area as

$$
\begin{aligned}
A & =A_{\text {fin }}+A_{d i v} \\
A_{\text {fin }} & =4 \int d^{2} z\left(e^{2 \alpha}-\sqrt{p \bar{p}}\right), \quad A_{d i v}=4 \int d^{2} z \sqrt{p \bar{p}} .
\end{aligned}
$$

Now by using the modified sinh-Gordon equation (2.16)

$$
\partial \bar{\partial} \alpha-e^{2 \alpha}+p \bar{p} e^{-2 \alpha}=0
$$

one can rewrite $A_{\text {fin }}$ into the form

$$
A_{\text {fin }}=2 \int d^{2} z\left(e^{2 \alpha}+p \bar{p} e^{-2 \alpha}-2 \sqrt{p \bar{p}}+\partial \bar{\partial} \alpha\right) .
$$

The contribution from the total derivative term $\partial \bar{\partial} \alpha$ comes solely from the "boundaries", which in our case consist of infinitesimal circles around the vertex insertion points and a large circle at infinity. For the contributions from the singularities, we may use (3.2) 
to replace $\partial \bar{\partial} \alpha$ by $\frac{1}{4} \partial \bar{\partial} \ln (p \bar{p})$. Furthermore, from the behavior of $p \bar{p}$ at the singularity and the familiar formula $\partial \bar{\partial} \ln z \bar{z}=\pi \delta^{2}(z)$, the integral $\frac{1}{4} \int d^{2} z \partial \bar{\partial} \ln p \bar{p}$ simply yields $\pi / 2$ for each of the singularity. On the other hand, for large $z$, as will be discussed in Sec. 3.3.2, $p(z)$ goes as $\sim 1 / z^{4}$, which again leads to the behavior $\alpha \simeq(1 / 4) \ln p \bar{p}$ from (3.5). Using the Stokes theorem the contribution of $\frac{1}{4} \int d^{2} z \partial \bar{\partial} \ln p \bar{p}$ from this region yields $-\pi$. Combining, for $N$-point correlation functions $A_{\text {fin }}$ can be written as

$$
\begin{aligned}
& A_{\text {fin }}=2 A_{\text {reg }}+\pi(N-2), \\
& A_{\text {reg }} \equiv \int d^{2} z\left(e^{2 \alpha}+p \bar{p} e^{-2 \alpha}-2 \sqrt{p \bar{p}}\right),
\end{aligned}
$$

where we defined $A_{\text {reg }}$, to be called the "regularized area". This will be the quantity to be evaluated in the remainder of this section. The divergent part $A_{d i v}$ will be discussed briefly in Sec.4, where we argue that when combined with the contribution of the vertex operators it will give a finite result.

\subsection{Comparison with the gluon scattering problem}

Before proceeding to the actual evaluation of the regularized area, it should be helpful to quickly review the salient features of the calculation of the corresponding quantity in the case of the gluon scattering amplitudes given in [13] and describe the similarity to and the difference from the computation that we are going to perform.

In [13], the momenta of the massless gluons were mapped by T-duality to the segments of a null polygonal Wilson loop and the scattering amplitude of such gluons is given by the area of the minimal surface bordered by that loop. For a simple configuration of gluon momenta, the loop can be taken to lie in a $R^{1,1}$ subspace of $R^{1,3}$ and the relevant minimal surface is contained in $A d S_{3}$. Although such an area is divergent reflecting the infrared divergence of the massless on-shell amplitude, one can extract the important finite part, called the remainder function, by regularizing the area as in (3.8). As it is difficult to construct the explicit solution of the minimal surface for case with more than four gluons, they utilized the framework of Pohlmeyer reduction and performed integrabilitybased analysis of the associated auxiliary linear problem to calculate the regularized area without the knowledge of such solutions.

In that analysis, the holomorphic function $p(z)$ which provides the input data of the gluons for the auxiliary linear problem behaves, in the case of $2 n$ gluons, as a polynomial in $z$ of degree $n-2$, viz,

$$
p(z)=z^{n-2}+\cdots,
$$


which has a singularity at infinity. Due to this singularity, the solutions to the auxiliary linear problems exhibit the so-called Stokes phenomena. Namely, the solutions change their asymptotic forms at infinity discontinuously as they cross a set of lines called Stokes lines. These lines partition the whole complex plane into several Stokes sectors. In each Stokes sector, labeled by $i$, there are two solutions $b_{i}$ and $s_{i}$, which are called "big" and "small" according as they increase or decrease asymptotically at infinity. It is extremely important to identify the small solution since it is uniquely defined contrary to the big solution which can contain a multiple of small solution and hence is ambiguous. It was shown that the essential information is carried by the $S L(2, C)$-invariant products $\left\langle s_{j}, s_{k}\right\rangle$ of these small solutions and they are related to each other through a system of functional equations called T-system, which depends on the spectral parameter $\xi$ :

$$
T_{j}\left(e^{+i \pi / 2} \xi\right) T_{j}\left(e^{-i \pi / 2} \xi\right)=T_{j-1} T_{j+1}+1, \quad T_{j} \equiv\left\langle s_{0}, s_{j+1}\right\rangle\left(e^{-i(j+1) \pi / 2} \xi\right) .
$$

On the other hand, with the aid of the Riemann bilinear identity, the regularized area of interest can be expressed in terms of certain boundary contour integrals on a bordered double cover of the worldsheet, where the important function defined by

$$
\Lambda(z) \equiv \int^{z} \sqrt{p\left(z^{\prime}\right)} d z^{\prime}
$$

is single-valued. The crucial fact is that these contour integrals appear in the WKB expansions $^{6}$ of the $S L(2, C)$-invariant products $\left\langle s_{j}, s_{k}\right\rangle$ discussed above. Therefore, by solving the $T$-system equations, one can compute the value of the regularized area.

As we shall explain in detail in the subsequent subsections, the procedure for the computation of the regularized area relevant to the three point function will be quite similar to what we described above. There are, however, some differences which require nontrivial modifications. Below let us preview them by comparing with the gluon scattering computation sketched above.

For the gluon scattering, the worldsheet is of the disk topology as it is an open string process. The boundary of the disk, however, is not directly visible in the actual calculation as it is mapped to a point at infinity. Its effect is encoded in the singularity of $p(z)$ at infinity. In contrast, in the case of three point functions of closed strings, the worldsheet is basically a sphere with the vertex operators inserted at three points $z_{i}$ and their effect is reflected in the singularities of $p(z)$ of the form ${ }^{7}$

$$
p(z) \stackrel{z \rightarrow z_{i}}{\sim} \frac{\delta_{i}^{2}}{\left(z-z_{i}\right)^{2}} .
$$

\footnotetext{
${ }^{6}$ Following [14] we shall refer to the expansion in powers of $\xi$ or $1 / \xi$ as WKB expansions.

${ }^{7}$ For LSGKP string, $\delta_{i}=i \kappa_{i} / 2$, as shown in $(2.45)$.
} 
These double pole singularities produce two main modifications in the calculation of the regularized area.

One significant modification is that one needs to generalize the Riemann bilinear identity in order to express the area in terms of contour integrals. In contrast to the case of the gluon scattering, due to the double pole singularities of $p(z)$ the function $\Lambda(z)$ acquires logarithmic branch cuts, on top of the usual square root cuts which are also present for the gluon scattering. The existence of such logarithmic branch cuts invalidates the application of the standard Riemann bilinear identity and one needs to derive an appropriate generalization to deal with this new situation.

The other modification stems from the difference of the way the information of the external states is encoded. In place of the behavior of the solution in different Stokes sectors in the case of the gluon scattering problem, such information is carried by the so called monodromy data for the solutions of the auxiliary linear problem . As will be explained more fully in subsection 3.4, a basis of solutions $\left(\eta_{1}, \eta_{2}\right)$ of the auxiliary linear problem gets transformed into a different basis $\left(\eta_{1}^{\prime}, \eta_{2}^{\prime}\right)$ by a monodromy matrix $M$ when analytically continued around each pole:

$$
\left(\begin{array}{l}
\eta_{1}^{\prime} \\
\eta_{2}^{\prime}
\end{array}\right)=M\left(\begin{array}{l}
\eta_{1} \\
\eta_{2}
\end{array}\right)
$$

It will be shown that the contour integrals which appear in the generalized Riemann bilinear identity can be expressed in terms of the $S L(2, C)$ invariant products between the eigenvectors of the monodromy matrices, which in turn can be expressed in terms of the parameters $\delta_{i}$ appearing in (3.12) from the properties of the monodromy matrices. This process actually corresponds to the solution of the T-system. To actually evaluate the $S L(2, C)$ invariant products we will need to make use of the notion of the small and big solutions and their WKB expansions, as in the case of the gluon scattering problem. In particular it will be important to find the precise relation between the $S L(2, C)$ products between the eigenfunctions of the monodromy matrices and those between the small solutions.

Having explained the relation to the gluon scattering problem, we now proceed to the actual computation of the regularized area relevant to the three point functions.

\subsection{Generalization of Riemann bilinear identity}

As in the case of the gluon scattering problem, we wish to reexpress the regularized area in terms of certain contour integrals. For the gluon scattering, this was made possible with the aid of the Riemann bilinear identity, which is essentially the familiar Stokes theorem 
applied to a Riemann surface with finite genus. Unfortunately, in the present case the usual Riemann bilinear identity is not directly applicable due to double pole singularities of $p(z)$. The presence of these singularities leads to logarithmic branch cuts in addition to the square root cuts, which must be properly treated.

In this subsection, we will derive an appropriate generalization of the Riemann bilinear identity, which is applicable in the presence of any number of singularities. Although we mainly consider, in this paper, three point functions of the large spin limit of the GKP strings, the identity we derive can be applied to $N$ point functions of general operators.

\subsubsection{From surface integrals to boundary contour integrals}

As the first step, we rewrite $A_{\text {reg }}$ defined in (3.8) as an integral over the product of two functions of the form

$$
A_{\text {reg }}=\int d^{2} z \lambda u
$$

where $\lambda$ and $u$ are given by

$$
\begin{aligned}
& \lambda=\sqrt{p}, \quad u=2 \sqrt{\bar{p}}(\cosh 2 \hat{\alpha}-1), \\
& \hat{\alpha} \equiv \alpha-\frac{1}{4} \ln p \bar{p} .
\end{aligned}
$$

It is easy to show that one can construct a closed 1-form of the form $u d \bar{z}+v d z$ with the help of a function $v$ given by

$$
v=\frac{1}{\sqrt{p}}(\partial \hat{\alpha})^{2} .
$$

Since $\lambda$ is holomorphic and $u d \bar{z}+v d z$ is closed, (3.14) can be reexpressed as

$$
\int d^{2} z \lambda u=\frac{i}{2} \int \lambda d z \wedge(u d \bar{z}+v d z) .
$$

Now due to the presence of the square root branch cuts, it will be convenient to consider a double cover of the worldsheet, to be denoted by $D$, with appropriate boundary $\partial D$, which will be specified more explicitly later. To distiguish the points and contours on the first and the second sheet clearly, we will put overhats on quantities on the second sheet. $D$ and $\partial D$ are defined so that the function

$$
\Lambda(z)=\int_{z_{0}}^{z} \lambda\left(z^{\prime}\right) d z^{\prime}
$$

is singled-valued and the functions $\lambda, u$ and $v$ have no singularities on $D$. The end point $z_{0}$ of the integral can be chosen arbitrarily on $D$ but in the following discussion we shall 
take $z_{0}$ to be on the first sheet. The corresponding point on the second sheet is denoted by $\widehat{z}_{0}$.

With this setting, we can convert the area integral (3.14) into a boundary contour integral in the following way ${ }^{8}$.

$$
A_{\text {reg }}=\frac{i}{4} \int_{D} d \Lambda \wedge(u d \bar{z}+v d z)=\frac{i}{4} \int_{D} d(\Lambda(u d \bar{z}+v d z))=-\frac{i}{4} \int_{\partial D} \Lambda(u d \bar{z}+v d z) .
$$

To proceed further we now specify $D$ and $\partial D$ explicitly for the worldsheet relevant to $N$ point functions for various $N$.

\subsubsection{Double cover of the worldsheet for $\mathrm{N}$ point functions}

What we need to know is the structure of the singularities and the zeros of $p(z)$. In the case of $N$ point function, there are $N$ double poles at $z_{i}$ for $i=1,2, \ldots, N$, where $p(z)$ behaves like

$$
p(z) \stackrel{z \rightarrow z_{i}}{\sim} \frac{\delta_{i}^{2}}{\left(z-z_{i}\right)^{2}}
$$

This means that $p(z)$ is of the form

$$
p(z)=\frac{f(z)}{\left(z-z_{1}\right)^{2} \cdots\left(z-z_{N}\right)^{2}},
$$

where $f(z)$ is a polynomial with the property $f\left(z_{i}\right)=\delta_{i}^{2} \prod_{j=1(\neq i)}^{N}\left(z_{i}-z_{j}\right)^{2}$. An important requirement is that there is no singularity at infinity. Since $p(z)$ is a $(2,0)$ form, this means that, by making a conformal transformation $z \rightarrow 1 / z,\left(1 / z^{4}\right) p(1 / z)$ should be nonsingular at $z=0$. This in turn is equivalent to the requirement $p(z) \sim \mathcal{O}\left(1 / z^{4}\right)$ for large $z$. From (3.22) we see that $f(z)$ is a polynomial in $z$ of order $2 N-4$ and hence $p(z)$ generically has $2 N-4$ zeros. We have thus found that the function $\Lambda(z)$ defined in (3.19) has $N$ logarithmic branch cuts running from the singularities and $N-2$ square root cuts connecting the $2 N-4$ zeros of $p(z)$.

With this knowledge, let us describe the appropriate domain $D$ and its boundary $\partial D$ for the cases of two, three and multi point functions.

First, in the case of a two point function, $\Lambda$ has one logarithmic branch cut which connects the two singularities and no square root cut. Therefore, we should choose $\partial D$ as a contour encircling two singularities and the log branch cut and take $D$ to be the domain outside, as in figure 3.2.

\footnotetext{
${ }^{8}$ Compared to the previous version, the sign of the last term has been reversed, in order to be consistent with the directions of the contours depicted in the figures. This resulted in obvious overall sign changes for various equations in this subsection.
} 


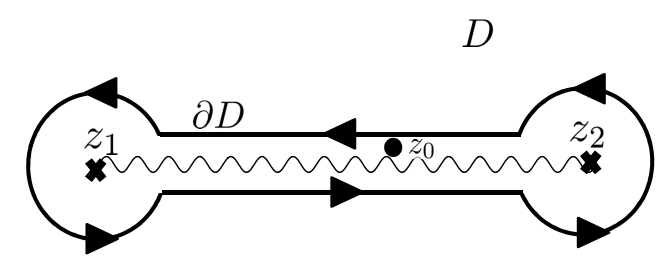

Figure 3.2: $D$ and $\partial D$ for a two point function. The wavy line represents the logarithmic cut for the function $\Lambda$.

Next consider the case of a three point function. For later purpose, let us display the explicit form of $p(z)$, which is completely fixed in terms of $\delta_{i}$ alone:

$$
\begin{aligned}
p(z) & =\left(\frac{\delta_{1}^{2} z_{12} z_{13}}{z-z_{1}}+\frac{\delta_{2}^{2} z_{21} z_{23}}{z-z_{2}}+\frac{\delta_{3}^{2} z_{31} z_{32}}{z-z_{3}}\right) \frac{1}{\left(z-z_{1}\right)\left(z-z_{2}\right)\left(z-z_{3}\right)} \\
z_{i j} & \equiv z_{i}-z_{j} .
\end{aligned}
$$

In this case, $\Lambda$ has three logarithmic branch cuts running out from the singularities and one square root branch cut. We choose the positions of logarithmic branch cuts so that they end at a single point on each sheet. For later convenience, on the first sheet we choose this point to be $z_{0}$, which is the initial point of the integral in the definition of $\Lambda$ (3.19), while on the second sheet the point is chosen to be $\widehat{z}_{0}$, right below $z_{0}$. Then, $D$ and $\partial D$ can be chosen as depicted in figure 3.3 .
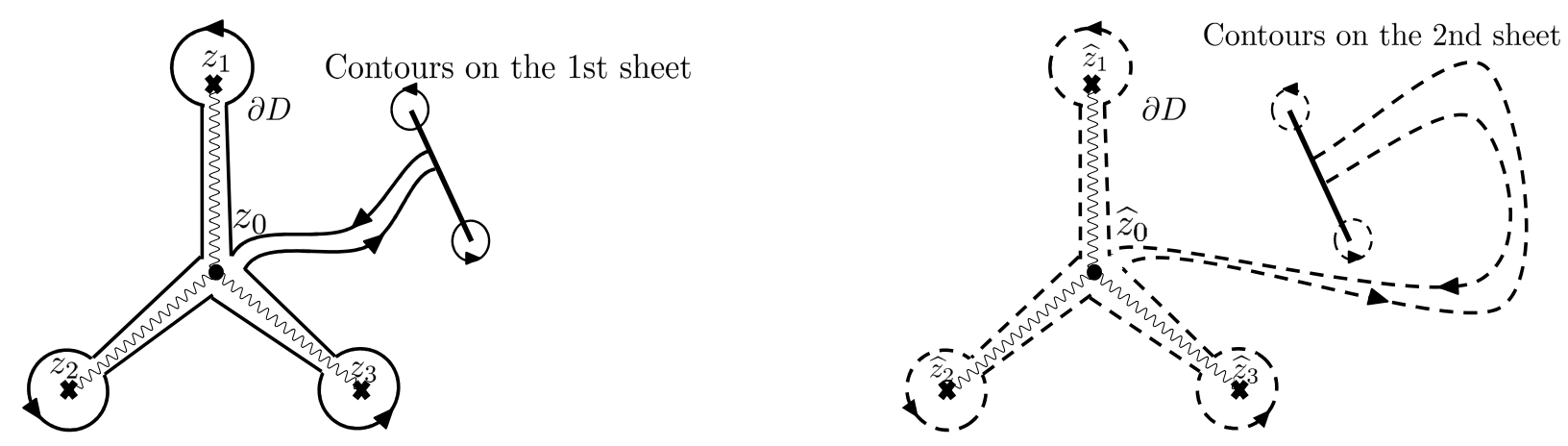

Figure 3.3: $D$ and $\partial D$ for a three point function. Contours on the first sheet are drawn as solid lines on the left, while the contours on the second sheet are shown as dashed lines on the right.

Finally, for the case of $N$ point function with $N>3$, there are more logarithmic as well as square root cuts. The basic idea, however, is the same. As in the case of the three point function, we choose the positions of logarithmic cuts so that they all end at $z_{0}$. Then $D$ and $\partial D$ are taken as shown in figure 3.4 . 

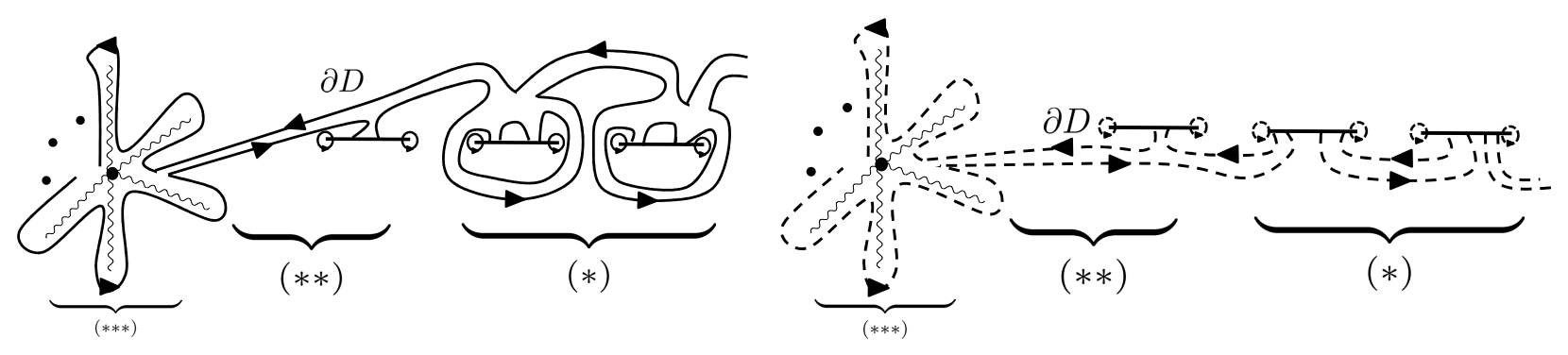

Figure 3.4: $D$ and $\partial D$ for a multi-point function

\subsubsection{Derivation of the generalized Riemann bilinear identity}

With the preparation above, we now derive a generalization of the Riemann bilinear identity. Basically, what we need to do is to evaluate the contributions from all the portions of the boundary $\partial D$, both in the first and the second sheet, shown in figure 3.4. We will do this by dividing $\partial D$ into several portions.

First we focus on the part consisting of contours which connect or encircle the square root branch cuts, forming the right half on each sheet of the figure $3.4,(*)$, and redrawn in a slightly different fashion in figure 3.5. As one can recognize immediately, they are precisely the contours which appear in the standard Riemann bilinear identity and hence the contribution can be written as

$$
\frac{i}{4}\left(\sum_{i} \oint_{a_{i}} \lambda d z \oint_{b_{i}}(u d \bar{z}+v d z)-\oint_{b_{i}} \lambda d z \oint_{a_{i}}(u d \bar{z}+v d z)\right),
$$

where the cycles $\left(a_{i}, b_{i}\right)$ are defined as in figure 3.5. This type of contribution, however,

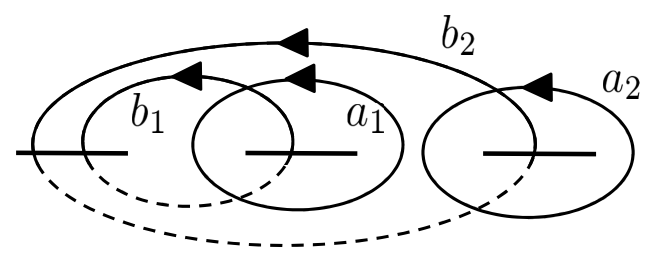

Figure 3.5: Type of contours, consisting of cycles $\left(a_{i}, b_{i}\right)$, for which the standard Riemann bilinear identity is applicable.

is absent for three point functions since there is only one square root cut.

Next, we consider the portion which connects the leftmost square root cut shown in figure 3.4 and the point $z_{0}$ located at the junction of the logarithmic cuts $((* *)$ in figure 3.4). Along this line the quantity $u d \bar{z}+v d z$ is single-valued but the function $\Lambda$ has different values along the two opposite sides (depicted by double lines in figure 3.4). The difference 
of these values is given by the integral of $\Lambda$ along the contour which connects these two sides in $D$, namely the one going around the logarithmic cuts. Thus, the contribution can be written as

$$
\frac{i}{4} \oint_{C} \lambda d z \oint_{d}(u d \bar{z}+v d z)
$$

where the contour $d$ and $C$ are shown in figure 3.6. The contribution from $\hat{d}$ on the second sheet can be obtained by substituting $d$ and $C$ in (3.25) with $\widehat{d}$ and $\hat{C}$. This turns out to be the same as to (3.25) since the only difference between two sheets is the sign of $\lambda d z$ and $u d \bar{z}+v d z$. Therefore, the net contribution is

$$
\frac{i}{2} \oint_{C} \lambda d z \oint_{d}(u d \bar{z}+v d z)
$$

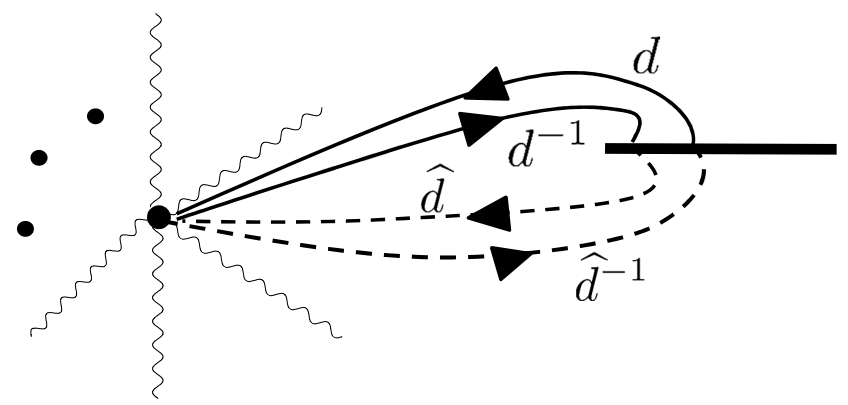

(a)

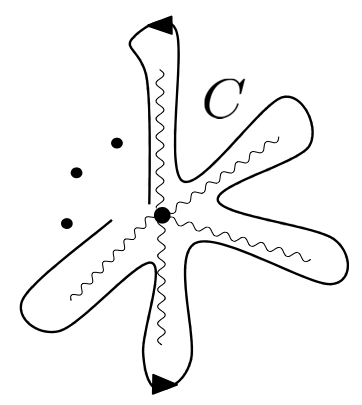

(b)

Figure 3.6: Definitions of the contours $d, d^{-1}, \hat{d}, \hat{d}^{-1}$ and $C . d^{-1}$ denotes a path parallel to $d$ but running in opposite direction. Hatted paths run on the second sheet.

Thirdly, we consider the contribution from contours which encircle the zeros of $p(z)$ as shown in figure 3.7. This contribution comes from singularities of $v=\frac{1}{\sqrt{p}}(\partial \hat{\alpha})^{2}$ at zeros of $p(z)$. In the vicinity of a zero of $p(z), v$ can be approximated by

$$
v=\frac{1}{\sqrt{p}}(\partial \hat{\alpha})^{2} \sim \frac{1}{\tilde{c} \sqrt{z-\tilde{z}}}\left(\partial \alpha-\frac{1}{4(z-\tilde{z})}\right)^{2},
$$

where $\tilde{z}$ is the position of the zero and $\tilde{c}$ is the constant which we do not need to specify here. Since $\alpha$ is not singular at zeros, the leading singularity around the zero is given by

$$
\frac{1}{16 \tilde{c}(z-\tilde{z})^{5 / 2}} \text {. }
$$

It turns out that this leading part gives a finite contribution as we show below, while the contributions from the sub-leading terms vanish. The integral on the contour $E$ encircling 
$\tilde{z}$ (see figure 3.7) can be evaluated as

$$
\begin{aligned}
\frac{i}{4} \int_{E} \Lambda(u d \bar{z}+v d z) & =\frac{i \Lambda(\tilde{z})}{4} \oint_{E} d z \frac{1}{16 \tilde{c}(z-\tilde{z})^{5 / 2}}+\frac{i}{4} \oint_{E} d z \frac{1}{16(z-\tilde{z})^{5 / 2}} \int_{\tilde{z}}^{z} d z^{\prime}\left(z^{\prime}-\tilde{z}\right)^{1 / 2} \\
& =-\frac{\pi}{24}
\end{aligned}
$$

where the first term, which contains $\tilde{c}$, vanishes upon performing the integration. Since all $2 N-4$ zeros give the same result, the net contribution is a constant which depends on $N$ :

$$
\frac{\pi}{12}(N-2)
$$
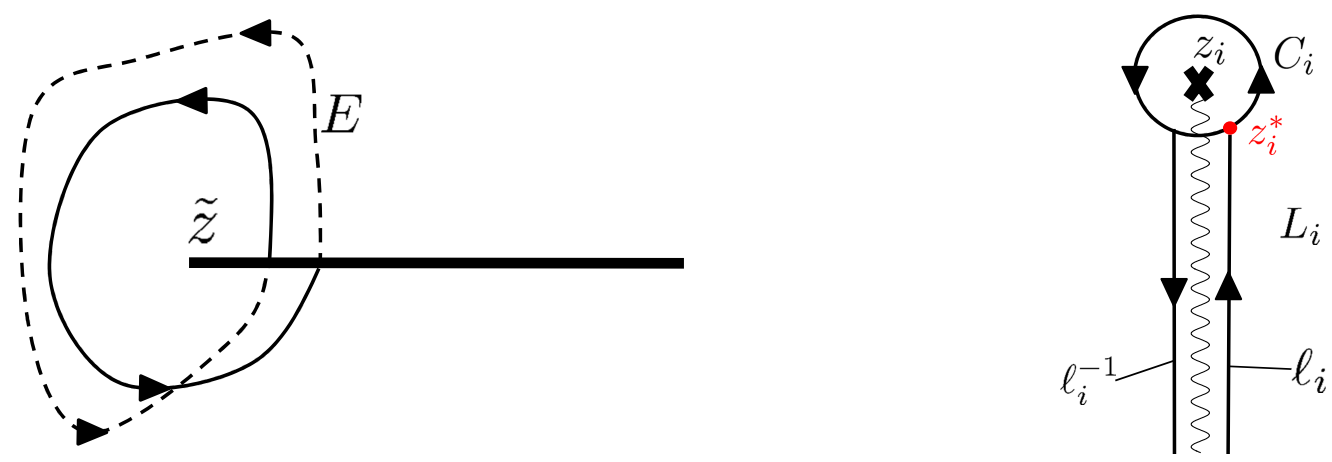

Figure 3.7: Contours encircling a zero of $p(z) \quad$ Figure 3.8: Contours $C_{i}, L_{i}$ and the point $z_{i}^{*}$.

The remaining contribution is from the contour encircling the logarithmic cuts $((* * *)$ in figure 3.4). As a contour this is the same as $C$ used in (3.24). There it appeared effectively to express the difference of the values of $\Lambda$ along $d$ and $d^{-1}$. Here, on the other hand, it is a genuine portion of $\partial D$ along which to compute the integral (3.20).

To evaluate this contribution, it is convenient to decompose $C$ into three portions $C_{1}+L_{1}, C_{2}+L_{2}, C_{3}+L_{3}$, where $C_{i}$ denotes a small circle around $z_{i}$ and $L_{i}$ consists of a line $l_{i}$ starting from $z_{0}$ and ending on $z_{i}$ and the return path $l_{i}^{-1}$ from $z_{i}$ to $z_{0}$ in opposite direction. (See figure 3.8.) In what follows, we will describe the contributions from the above contours on the first sheet. The corresponding contributions from the second sheet can be obtained similarly, as will be briefly indicated later. Consider first the contribution from $L_{i}$. In exactly the same way as in the previous analysis of the contour $d$, the difference of the value of $\Lambda$ along $\ell_{i}$ and $\ell_{i}^{-1}$ is represented by the integral $\oint_{C_{i}} \lambda d z$ and hence the net contribution from $L_{i}$ can be evaluated as

$$
\frac{i}{4} \oint_{C_{i}} \lambda d z \int_{\ell_{i}}(u d \bar{z}+v d z)
$$


Next, to evaluate the contribution from the circle $C_{i}$, it is convenient to re-express $\Lambda(z)$ around $z_{i}$ as

$$
\Lambda(z)=\Lambda\left(z_{i}^{*}\right)+\int_{z_{i}^{*}}^{z} \lambda d z^{\prime}
$$

where $z_{i}^{*}$ is the initial point of the contour $C_{i}$ as shown in figure 3.8. Then, the contribution from $C_{i}$ can be evaluated as

$$
-\frac{i}{4} \oint_{C_{i}} \Lambda(u d \bar{z}+v d z)=-\frac{i}{4} \Lambda\left(z_{i}^{*}\right) \oint_{C_{i}}(u d \bar{z}+v d z)-\frac{i}{4} \oint_{C_{i}}(u d \bar{z}+v d z) \int_{z_{i}^{*}}^{z} \lambda d z^{\prime} .
$$

Now note that $\Lambda\left(z_{i}^{*}\right)$ can be written in the following way, starting from its value at $z_{0}$ and integrating along the contour from $z_{0}$ to $z_{i}^{*}$ :

$$
\Lambda\left(z_{i}^{*}\right)=\Lambda\left(z_{0}\right)+\int_{\ell_{i}} \lambda d z+\sum_{j<i} \oint_{C_{j}} \lambda d z
$$

The last term signifies the contributions from around $C_{j}$ 's which are encountered on the way from $z_{0}$ to $z_{i}^{*}$. Using this expression in (3.33) and summing over the contributions from all $C_{i}$ 's, we arrive at the following simpler expression:

$$
\begin{aligned}
& -\frac{i}{4} \Lambda\left(z_{0}\right) \oint_{\sum_{i} C_{i}(=C)}(u d \bar{z}+v d z)-\frac{i}{4} \sum_{i} \int_{\ell_{i}} \lambda d z \oint_{C_{i}}(u d \bar{z}+v d z) \\
& -\frac{i}{8} \sum_{i \neq j} \oint_{C_{i}} \lambda d z \oint_{C_{j}}(u d \bar{z}+v d z)-\frac{i}{4} \sum_{i} \oint_{C_{i}}(u d \bar{z}+v d z) \int_{z_{i}^{*}}^{z} \lambda d z^{\prime} .
\end{aligned}
$$

So far, we have only considered the contribution from the first sheet. However, the expression we obtained (3.31), (3.35) can be easily generalized to the contours on the second sheet, since the only difference is the sign of $\lambda d z$ and $u d \bar{z}+v d z$. Thus, while (3.31) will not be changed on the second sheet, the result (3.35) will be modified as

$$
\begin{aligned}
& \frac{i}{4} \Lambda\left(\widehat{z}_{0}\right) \oint_{C}(u d \bar{z}+v d z)-\frac{i}{4} \sum_{i} \int_{\ell_{i}} \lambda d z \oint_{C_{i}}(u d \bar{z}+v d z) \\
& -\frac{i}{8} \sum_{i \neq j} \oint_{C_{i}} \lambda d z \oint_{C_{j}}(u d \bar{z}+v d z)-\frac{i}{4} \sum_{i} \oint_{C_{i}}(u d \bar{z}+v d z) \int_{z_{i}^{*}}^{z} \lambda d z^{\prime} .
\end{aligned}
$$

This completes the evaluation of contributions coming from various parts of the contour along $\partial D$. Gathering all the results obtained above, namely (3.24), (3.26),(3.30), (3.31), (3.35) and (3.36), we can now write down the generalized Riemann bilinear identity, which expresses the integral (3.14) over the worldsheet in terms of line integrals. To exhibit it compactly, let us introduce the following notion:

$$
\llbracket A, B \rrbracket \equiv \int_{A} \lambda d z \int_{B}(u d \bar{z}+v d z)
$$


Then, the generalized identity takes the form ${ }^{9}$

$$
\begin{aligned}
\int d^{2} z \lambda u & =\frac{\pi}{12}(N-2)+\frac{i}{4}\left(2(\llbracket C, d \rrbracket-\llbracket d, C \rrbracket)+\sum_{i} \llbracket C_{i}, C_{i} \rrbracket+\sum_{i}\left(\llbracket a_{i}, b_{i} \rrbracket-\llbracket b_{i}, a_{i} \rrbracket\right)\right. \\
& \left.+2 \sum_{j}\left(\llbracket C_{j}, \ell_{j} \rrbracket-\llbracket \ell_{j}, C_{j} \rrbracket\right)\right)-\frac{i}{2} \sum_{i} \oint_{C_{i}}(u d \bar{z}+v d z) \int_{z_{i}^{*}}^{z} \lambda d z^{\prime} .
\end{aligned}
$$

Note the following feature: Except for the terms in the last sum, each term on the right hand side is of the form of a product of two contour integrals, just like the standard bilinear identity. Unfortunately, the last sum consists of undecoupled double integrals which apparently have no simple expressions in terms of some contour integrals of $\lambda d z$ and/or $u d \bar{z}+v d z$.

\subsubsection{Simplification for the LSGKP string}

Because the contributions from different types of branch cuts are properly taken into account, the generalized Riemann bilinear identity derived above appears to be somewhat complicated in form. However, when applied to the case of LSGKP strings, the identity simplifies significantly. The main reason is that the function $\hat{\alpha}$, which appears in the definitions of $u$ and $v$, vanishes together with its derivative $\partial \hat{\alpha}$ near each singularity $z_{i}$. Therefore $u d \bar{z}+v d z$ vanishes at these points and hence all the terms which contain the factor $\oint_{C_{i}}(u d \bar{z}+v d z)$ vanish. The last term of (3.38), which is still a double integral, also vanishes since the integral over $z$, which involves the factor $u d \bar{z}+v d z$, is around $C_{i}$. Thus the identity (3.38) reduces, for LSGKP strings, to

$$
\int d^{2} z \lambda u=\frac{\pi}{12}(N-2)+\frac{i}{4}\left(2 \llbracket C, d \rrbracket+\sum_{i}\left(\llbracket a_{i}, b_{i} \rrbracket-\llbracket b_{i}, a_{i} \rrbracket\right)+2 \sum_{j} \llbracket C_{j}, \ell_{j} \rrbracket\right) .
$$

Moreover, in the case of three point functions, further simplification of the formula occurs as there is only one square root cut in the double cover of the worldsheet. This means that the terms involving the contour integrals over the cycles $a_{i}$ and $b_{i}$ are absent and the identity becomes

$$
\int d^{2} z \lambda u=\frac{\pi}{12}+\frac{i}{2}\left(\llbracket C, d \rrbracket+\sum_{j} \llbracket C_{j}, \ell_{j} \rrbracket\right)
$$

9 The equation (3.38) in the previous version of this paper contained an error due to an incorrect choice of the contour. This is fixed in the equation below. See Erratum [46] for further explanation of the correction. 

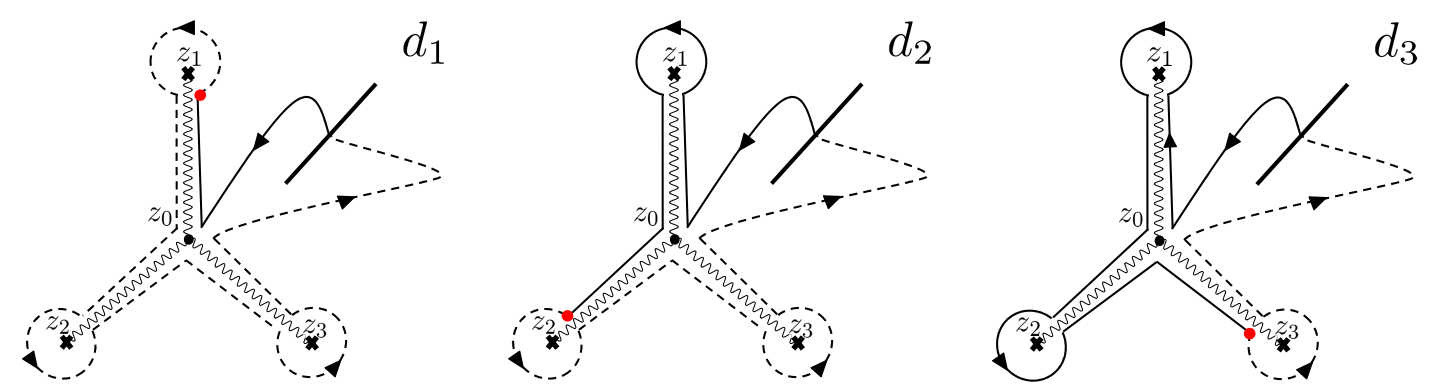

Figure 3.9: Definitions of the contours $d_{1}, d_{2}$ and $d_{3}$.

For later convenience we reexpress (3.40) as

$$
\int d^{2} z \lambda u=\frac{\pi}{12}+\frac{i}{4}\left(\sum_{j} \llbracket C_{j}, d_{j} \rrbracket\right),
$$

where $d_{j}$ is a contour which starts from $\widehat{z}_{j}$, goes around the logarithmic cuts counterclockwise and reaches $z_{j}$ as shown in figure 3.9. To see the equality of (3.40) and (3.41), simply express $C$ and $d_{j}$ 's in terms of "components" such as $C_{i}, \hat{C}_{i}, l_{i}, \hat{l}_{i}$ and use the fact that $\int_{C_{i}}(u d \bar{z}+v d z)$ vanishes in the case of GKP strings.

\subsection{Monodromy matrices and eigenfunctions for auxiliary linear problems}

In order to evaluate the regularized area using the generalized Riemann bilinear identity just derived, we need to know the behavior of the solutions of the auxiliary linear problem in which the function $p(z)$ has three double pole singularities. In this subsection, we will extract some important information from the so-called monodromy properties. As the auxiliary linear problem is a system of first order linear differential equations for a 2-component vector function, there are two independent solutions. Let $\left(\eta_{1}, \eta_{2}\right)$ be a particular basis of such solutions. Then, as one analytically continues them once around a singularity, they get transformed into a linear combination in the manner

$$
\left(\begin{array}{l}
\eta_{1}^{\prime} \\
\eta_{2}^{\prime}
\end{array}\right)=M\left(\begin{array}{l}
\eta_{1} \\
\eta_{2}
\end{array}\right)
$$

where the $2 \times 2$ matrix $M$ is called the monodromy matrix around that singularity. In what follows we will determine the three monodromy matrices $M_{i}$ for the three singularities, find their eigenvectors and compute the $S L(2, C)$ invariant products between them, which will play crucial roles in the evaluation of the contour integrals appearing in (3.41). 


\subsubsection{Eigenvalues of monodromy matrices and "plus" and "minus" eigenvec- tors}

Since the monodromy matrices $M_{i}$ belong to $S L(2, C)$, each of them can be diagonalized in the form ${ }^{10}$

$$
\left(\begin{array}{cc}
e^{i \rho_{i}(\xi)} & 0 \\
0 & e^{-i \rho_{i}(\xi)}
\end{array}\right)
$$

The (logarithm of the) eigenvalues $\rho_{i}(\xi)$ carry the basis-independent information and will be referred to as the local monodromy data ${ }^{11}$. The dependence of $\rho_{i}(\xi)$ on the spectral parameter encodes the information of the (infinite number of ) conserved charges of the integrable system, which includes that of the global charges such as the AdS energy $E$ and the spin $S$ of the GKP solution. Therefore the information of the external string states is contained in $\rho_{i}(\xi)$ and the regularized area should be expressible in terms of these local monodromy data.

Let us now look at the structure of the eigenvectors of the monodromy matrices closely. Near each of the singularity $z_{i}$, the eigenvectors must behave like the external string state of interest. Since the LSGKP strings of our interest satisfy the relation $e^{2 \alpha}=\sqrt{p \bar{p}}$, the eigenvectors near $z_{i}$ take the form (see (2.50))

$$
\psi \sim \exp \left[ \pm\left(\frac{1}{\xi} \int \sqrt{p(z)} d z+\xi \int \sqrt{\bar{p}(\bar{z})} d \bar{z}\right)\right]
$$

Here and in what follows, we define $\sqrt{p(z)}$ and $\sqrt{\bar{p}(\bar{z})}$ as the values on the first sheet. Then, we can distinguish the two independent eigenfunctions of $M_{i}$ by the behavior at the point $z_{i}$. More precisely, we call the eigenvector $i_{ \pm}$("plus" and "minus" solution ), if it behaves asymptotically like

$$
\sim \exp \left[ \pm\left(\frac{1}{\xi} \int \sqrt{p(z)} d z+\xi \int \sqrt{\bar{p}(\bar{z})} d \bar{z}\right)\right]
$$

at $z_{i}$. For later convenience, we impose the normalization condition

$$
\left\langle i_{+}, i_{-}\right\rangle=1
$$

Note that there still remains a freedom of rescaling $i_{+}$and $i_{-}$oppositely, namely, $i_{+} \rightarrow$ $A i_{+}, i_{-} \rightarrow A^{-1} i_{-}$. In this description the eigenvalue of the monodromy $\rho_{i}(\xi)$ can be defined by the phase factors acquired by $i_{+}$and $i_{-}$

$$
i_{+}^{\prime}=e^{i \rho_{i}(\xi)} i_{+}, \quad i_{-}^{\prime}=e^{-i \rho_{i}(\xi)} i_{-},
$$

\footnotetext{
${ }^{10}$ It is generically not possible to diagonalize all the monodromy matrices simultaneously.

11 The quantity $\rho_{i}$ is called "pseudo momentum" and often denoted by $p_{i}$. In this paper, we use a different letter to distinguish it from the function $p(z)$.
} 
where $i_{+}^{\prime}, i_{-}^{\prime}$ are the analytic continuation of $i_{+}, i_{-}$along a path which encircles the singularity counterclockwise.

We should emphasize that the notion of "plus" and "minus" solutions introduced above is conceptually quite distinct from that of "small" and "big" solutions that appeared in the gluon scattering problem. "Small" and "big" refer to the corresponding behavior of the solution as one approaches the singularity (which in the gluon scattering problem corresponds to the segment of the polygon at infinity). Only the small solution is unambiguously defined since the big solution can contain a multiple of small solution. On the other hand, the plus and minus solutions are simply the convenient and unambiguous characterization of the two independent eigenvectors of the monodromy matrix. Later when we perform the WKB analysis of the auxiliary linear problem, we will need the notion of small and big solutions as well. The precise relation between the plus-minus solutions and the small solution will be of utmost importance when we compute the contour integrals in terms of the $S L(2, C)$ invariant products in section 3.6.

\subsubsection{Calculation of explicit forms of monodromy matrices}

Let us now determine the explicit form of the monodromy matrices $M_{i}$, up to conjugacy classes. Since we can diagonalize any one of $M_{i}$, we choose a basis $\left\{1_{+}, 1_{-}\right\}$, normalized as $\left\langle 1_{+}, 1_{-}\right\rangle=1$, such that $M_{1}$ is diagonal:

$$
M_{1}=\left(\begin{array}{cc}
e^{i \rho_{1}(\xi)} & 0 \\
0 & e^{-i \rho_{1}(\xi)}
\end{array}\right) .
$$

We then write $M_{2}$ in the form

$$
M_{2}=\left(\begin{array}{ll}
a & b \\
c & d
\end{array}\right)
$$

where, from the $S L(2, C)$ and the trace properties, the components $a$ to $d$ satisfy the following relations

$$
\begin{aligned}
a d-b c & =1, \\
a+d & =e^{i \rho_{2}(\xi)}+e^{-i \rho_{2}(\xi) .} .
\end{aligned}
$$

Since the monodromy around all the singularities must be trivial, we must have the relation

$$
M_{1} M_{2} M_{3}=1
$$

This determines $M_{3}$ as $M_{3}=\left(M_{1} M_{2}\right)^{-1}$ and taking the trace of this relation we get

$$
e^{i \rho_{1}(\xi)} a+e^{-i \rho_{1}(\xi)} d=e^{i \rho_{3}(\xi)}+e^{-i \rho_{3}(\xi)} .
$$


The relations (3.50), (3.51) and (3.53) provide three equations for four variables $a, b, c, d$. However, as $b$ and $c$ appear in (3.50) only in the product $b c$, we can completely solve for $a, d$ and $b c$ in terms of $\rho_{i}$. The result is

$$
\begin{aligned}
& a=\frac{\cos \rho_{3}-e^{-i \rho_{1}} \cos \rho_{2}}{i \sin \rho_{1}}, \quad d=\frac{e^{i \rho_{1}} \cos \rho_{2}-\cos \rho_{3}}{i \sin \rho_{1}}, \\
& b c=\frac{\cos ^{2} \rho_{1}+\cos ^{2} \rho_{2}+\cos ^{2} \rho_{3}-2 \cos \rho_{1} \cos \rho_{2} \cos \rho_{3}-1}{\sin ^{2} \rho_{1}} .
\end{aligned}
$$

\subsubsection{Eigenvectors of monodromy matrices}

Having found the form of the non-trivial monodromy matrices $M_{2}, M_{3}$, we now compute their eigenvectors by diagonalizing them. This amounts to expressing the eigenvectors $\left\{2_{+}, 2_{-}\right\}$and $\left\{3_{+}, 3_{-}\right\}$in terms of $\left\{1_{+}, 1_{-}\right\}$. It will allow us to compute various $S L(2, C)$ invariant products between them, which will play important roles later in the calculation of the regularized area.

First consider the eigenvectors $\left\{2_{+}, 2_{-}\right\}$of $M_{2}$. Using the expressions of $a, d$ and $b c$ obtained in (3.54) and (3.55), they can be computed straightforwardly as

$$
\begin{aligned}
& 2_{+} \propto\left(\begin{array}{c}
\frac{2 i}{\sin \rho_{1}}\left(\cos \left(\rho_{1}-\rho_{2}\right)-\cos \rho_{3}\right) \\
2 c
\end{array}\right), \\
& 2_{-} \propto\left(\begin{array}{c}
\frac{2 i}{\sin \rho_{1}}\left(\cos \left(\rho_{1}+\rho_{2}\right)-\cos \rho_{3}\right) \\
2 c
\end{array}\right) .
\end{aligned}
$$

Note that these expressions contain $c$, the undetermined component of $M_{2}$. This reflects the rescaling ambiguity still left in $\left\{1_{+}, 1_{-}\right\}$. If we further impose the normalization condition $\left\langle 2_{+}, 2_{-}\right\rangle=1$, the eigenvectors become

$$
\begin{aligned}
& 2_{+}=A_{2}\left(\begin{array}{c}
\frac{i}{\sin \rho_{1}}\left(\cos \left(\rho_{1}-\rho_{2}\right)-\cos \rho_{3}\right) \\
c
\end{array}\right), \\
& 2_{-}=\frac{1}{2 A_{2} \sin \rho_{2}}\left(\begin{array}{c}
\frac{1}{c \sin \rho_{1}}\left(\cos \left(\rho_{1}+\rho_{2}\right)-\cos \rho_{3}\right) \\
-i
\end{array}\right),
\end{aligned}
$$

where $A_{2}$ indicates the remaining scaling ambiguity for $\left\{2_{+}, 2_{-}\right\}$.

Next, consider the eigenvectors $\left\{3_{+}, 3_{-}\right\}$of the matrix $M_{3}$, which is given by

$$
\begin{aligned}
& M_{3}=M_{2}^{-1} M_{1}^{-1} \\
& =\left(\begin{array}{cc}
\frac{\cos \rho_{2}-e^{-i \rho_{1}} \cos \rho_{3}}{i \sin \rho_{1}} & -\frac{\cos ^{2} \rho_{1}+\cos ^{2} \rho_{2}+\cos ^{2} \rho_{3}-2 \cos \rho_{1} \cos \rho_{2} \cos \rho_{3}-1}{c \sin ^{2} \rho_{1}} e^{-i \rho_{1}} \\
-c e^{i \rho_{1}} & \frac{e^{2} \rho_{1} \cos \rho_{3}-\cos \rho_{2}}{i \sin \rho_{1}}
\end{array}\right) .
\end{aligned}
$$


With the imposition of the condition $\left\langle 3_{+}, 3_{-}\right\rangle=1$ the eigenvectors are obtained as

$$
\begin{aligned}
& 3_{+}=A_{3}\left(\begin{array}{c}
\frac{i e^{-i \rho_{1}}}{\sin \rho_{1}}\left(\cos \rho_{2}-\cos \left(\rho_{1}-\rho_{3}\right)\right) \\
c
\end{array}\right), \\
& 3_{-}=\frac{1}{2 A_{3} \sin \rho_{3}}\left(\begin{array}{c}
\frac{-1}{c \sin \rho_{1}}\left(\cos \rho_{2}-\cos \left(\rho_{1}+\rho_{3}\right)\right) \\
i e^{i \rho_{1}}
\end{array}\right),
\end{aligned}
$$

where again $A_{3}$ denotes an undetermined constant.

\subsubsection{SL(2,C) invariant products between different eigenvectors}

With the expressions of the eigenvectors obtained above, we can easily compute the $S L(2, C)$ invariant products between them. Those between the "plus" eigenvectors are evaluated as

$$
\begin{aligned}
& \left\langle 1_{+}, 2_{+}\right\rangle=c A_{2}, \\
& \left\langle 2_{+}, 3_{+}\right\rangle=c A_{2} A_{3}\left(e^{i\left(\rho_{3}-\rho_{1}\right)}-e^{-i \rho_{2}}\right), \\
& \left\langle 3_{+}, 1_{+}\right\rangle=-c A_{3} .
\end{aligned}
$$

On the other hand, the ones between the "minus" eigenvectors are given by

$$
\begin{aligned}
& \left\langle 1_{-}, 2_{-}\right\rangle=-\left(2 A_{2} c \sin \rho_{1} \sin \rho_{2}\right)^{-1}\left(\cos \left(\rho_{1}+\rho_{2}\right)-\cos \rho_{3}\right), \\
& \left\langle 2_{-}, 3_{-}\right\rangle=-\left(4 c A_{2} A_{3} \sin \rho_{2} \sin \rho_{3}\right)^{-1}\left(e^{i\left(\rho_{1}+\rho_{2}\right)}-e^{-i \rho_{3}}\right) \\
& \left\langle 3_{-}, 1_{-}\right\rangle=-\left(2 A_{3} c \sin \rho_{1} \sin \rho_{3}\right)^{-1}\left(\cos \rho_{2}-\cos \left(\rho_{1}+\rho_{3}\right)\right) .
\end{aligned}
$$

Lastly, the products between the "plus" and the "minus" eigenvectors are obtained as

$$
\begin{aligned}
\left\langle 1_{+}, 2_{-}\right\rangle & =-\frac{i}{2 A_{2} \sin \rho_{2}}, \\
\left\langle 1_{-}, 2_{+}\right\rangle & =-\frac{i A_{2}}{\sin \rho_{1}}\left(\cos \left(\rho_{1}-\rho_{2}\right)-\cos \rho_{3}\right), \\
\left\langle 2_{+}, 3_{-}\right\rangle & =-\frac{i A_{2}}{2 A_{3} \sin \rho_{3}}\left(e^{i\left(\rho_{1}-\rho_{2}\right)}-e^{-i \rho_{3}}\right), \\
\left\langle 2_{-}, 3_{+}\right\rangle & =-\frac{i A_{3}}{2 A_{2} \sin \rho_{2}}\left(e^{i\left(\rho_{3}-\rho_{1}\right)}-e^{i \rho_{2}}\right), \\
\left\langle 3_{+}, 1_{-}\right\rangle & =-\frac{i A_{3} e^{-i \rho_{1}}}{\sin \rho_{1}}\left(\cos \left(\rho_{1}-\rho_{3}\right)-\cos \rho_{2}\right), \\
\left\langle 3_{-}, 1_{+}\right\rangle & =-\frac{i e^{i \rho_{1}}}{2 A_{3} \sin \rho_{3}} .
\end{aligned}
$$

Several remarks are in order. 
- First, the constants $c, A_{2}$ and $A_{3}$, which are not yet fixed, may depend on the spectral parameter $\xi$ in unknown ways. This circumstance would give us trouble when we later make a WKB expansion in $\xi$ to extract the information of the contour integrals from the $S L(2, C)$ invariant products. Fortunately, this problem can be circumvented if we focus on certain combination of the products which do not depend on these constants. For instance the quantity

$$
\left\langle 1_{+}, 2_{+}\right\rangle\left\langle 1_{-}, 2_{-}\right\rangle=-\left(2 \sin \rho_{1} \sin \rho_{2}\right)^{-1}\left(\cos \left(\rho_{1}+\rho_{2}\right)-\cos \rho_{3}\right)
$$

is such a combination.

- Second, in computing the invariant products $\langle i, j\rangle$, which reflect the global structure of the solution, from the local monodromy data, we have not made explicit use of the $Z_{2}$ symmetry mentioned in subsection 2.1. As this symmetry stems from the Virasoro conditions, our method of analysis should be useful even when such conditions are not satisfied, for example for strings rotating in $S^{5}$ as well as in $A d S_{5}$.

\subsection{WKB analysis of the auxiliary linear problem}

In the previous subsection, we have computed the $S L(2, C)$ invariant products between the eigenvectors of the monodromy matrices in terms of the local monodromy data $\rho_{i}(\xi)$. These eigenvectors, called $i_{ \pm}$, are classified by the \pm signs in the exponent of these solutions. In order to make use of these invariant products in the computation of the regularized area, we must relate them to the contour integrals via WKB expansions of these quantities.

However, since the classification of the solutions as $i_{ \pm}$is not directly related to the WKB expansion, it will be more convenient to first introduce a different classification which is more closely connected to such an expansion. This is the notion of the "small" solution $s_{i}$ and the "big" solution $b_{i}$, associated to each singularity $z_{i}$. In the case of LSGKP strings, due to the prescribed behavior at the singularities, namely $e^{2 \alpha} \sim \sqrt{p \bar{p}}$, one of the two independent solutions of the auxiliary linear problem must decrease, while the other increases, as one approaches a singularity at $z_{i}$. The former is called the small solution $s_{i}$ and the latter the big solution $b_{i}$. Although such definitions of small and big solutions do not refer to the WKB expansion, they are actually closely tied to it since the leading term of the WKB expansion near $z_{i}$ tells us whether it is $s_{i}$ or $b_{i}$. The small solution is particularly important as it is uniquely determined. Contrarily, the big solution is ambiguous as it can contain a multiple of small solution.

In this subsection we shall develop the WKB expansion of the $S L(2, C)$ invariant products of small solutions $\left\langle s_{i}, s_{j}\right\rangle$ and observe that the same contour integrals that appear 
in the expression of the regularized area show up in that expansion. Later in subsection 3.6 , we will elucidate the relation between the $i_{ \pm}$eigenvectors and the small solutions $s_{i}$ and thereby connect the local monodromy data to these contour integrals.

\subsubsection{WKB expansions}

Before we begin the WKB expansion of the solutions of the auxiliary linear problem, we should make a remark on the validity of such an expansion. As it is a perturbative expansion in powers of $\xi$ (or $\xi^{-1}$ ), it is merely an asymptotic expansion and hence is known to be reliable only on a set of curves called the WKB curves [14]. The WKB curves are the ones on the complex plane which satisfy the equation

$$
\operatorname{Im}\left[\frac{1}{\xi} \sqrt{p} \frac{d z}{d t}\right]=0
$$

where $t$ parametrizes the curve. Along a WKB curve, the phase of the leading part of the WKB solution is constant and hence one can follow the change of the magnitude of the solution reliably. In what follows, we will always assume that WKB expansions are made along such WKB curves. For a generic value of $\xi$, a WKB curve starts from a singularity and ends at another singularity.

The auxiliary linear problem we need to solve was given in (2.32), namely

$$
\left(\partial+B_{z}(\xi)\right) \psi(\xi)=0, \quad\left(\bar{\partial}+B_{\bar{z}}(\xi)\right) \psi(\xi)=0
$$

where the $\xi$-dependent flat connections $B_{z}(\xi), B_{\bar{z}}(\xi)$ take the forms shown in $(2.20) \sim$ (2.22). As in the treatment of the LSGKP solution, one can simplify the equations by the gauge transformation discussed in (2.47), viz.,

$$
\psi=\left(\begin{array}{cc}
p^{-1 / 4} e^{\alpha / 2} & 0 \\
0 & p^{1 / 4} e^{-\alpha / 2}
\end{array}\right) \tilde{\psi}, \quad \tilde{\psi}=\left(\begin{array}{c}
\tilde{\psi}_{1} \\
\tilde{\psi}_{2}
\end{array}\right) .
$$

If we further use the variable $\hat{\alpha}=\alpha=\frac{1}{4} \ln p \bar{p}$ and switch to the worldsheet coordinate $w$ defined by $d w=\sqrt{p(z)} d z$, the equations for $\tilde{\psi}$ simplify to

$$
\begin{aligned}
& {\left[\partial_{w}+\frac{1}{\xi}\left(\begin{array}{cc}
0 & -1 \\
-1 & 0
\end{array}\right)+\left(\begin{array}{cc}
\partial_{w} \hat{\alpha} & 0 \\
0 & -\partial_{w} \hat{\alpha}
\end{array}\right)\right] \tilde{\psi}=0,} \\
& {\left[\bar{\partial}_{w}+\xi\left(\begin{array}{cc}
0 & -e^{-2 \hat{\alpha}} \\
-e^{2 \hat{\alpha}} & 0
\end{array}\right)\right] \tilde{\psi}=0 .}
\end{aligned}
$$

To solve these equations in powers of $\xi$, it is convenient to first analyze the second order equations for $\tilde{\psi}_{1}$, obtained by combining the above first-order equations, and then compute 
$\tilde{\psi}_{2}$ in terms of $\tilde{\psi}_{1}$. The equations for $\tilde{\psi}_{1}$ become

$$
\begin{aligned}
& \partial_{w}^{2} \tilde{\psi}_{1}-\frac{1}{\xi^{2}}\left(1+\xi^{2}\left(\left(\partial_{w} \hat{\alpha}\right)^{2}-\partial_{w}^{2} \hat{\alpha}\right)\right) \tilde{\psi}_{1}=0, \\
& \bar{\partial}_{w}^{2} \tilde{\psi}_{1}+2 \bar{\partial}_{w} \hat{\alpha} \bar{\partial}_{w} \tilde{\psi}_{1}-\xi^{2} \tilde{\psi}_{1}=0
\end{aligned}
$$

and $\tilde{\psi}_{2}$ are expressed in terms of $\tilde{\psi}_{1}$ in two ways as

$$
\begin{aligned}
\tilde{\psi}_{2} & =\xi\left(\partial_{w} \tilde{\psi}_{1}+\partial_{w} \hat{\alpha} \tilde{\psi}_{1}\right), \\
\tilde{\psi}_{2} & =\frac{1}{\xi} e^{2 \hat{\alpha}} \bar{\partial}_{w} \tilde{\psi}_{1} .
\end{aligned}
$$

We now make the WKB expansion of $\tilde{\psi}_{1}$ in powers of $\xi$ in the form

$$
\tilde{\psi}_{1}=\exp \left[\frac{S_{-1}}{\xi}+S_{0}+\xi S_{1}+\xi^{2} S_{2}+\cdots\right],
$$

and substitute this into the equations (3.80) and (3.81). Then at each order of $\xi$ we obtain equations involving $\partial_{w}$ and $\bar{\partial}_{w}$ derivatives and making use of the result of the previous order one can solve these equations successively. As the procedure is more or less straightforward, we will only sketch the computation at first two orders.

For the function $S_{-1}$ of the leading order, one obtains the following two equations

$$
\left(\partial_{w} S_{-1}\right)^{2}=1, \quad\left(\bar{\partial}_{w} S_{-1}\right)^{2}=0
$$

with the solutions

$$
S_{-1}= \pm w
$$

At the next order, we get the equations of the form

$$
\begin{aligned}
& \partial_{w}^{2} S_{-1}+2 \partial_{w} S_{-1} \partial_{w} S_{0}=0 \\
& \bar{\partial}_{w}^{2} S_{-1}+2 \bar{\partial}_{w} S_{-1} \bar{\partial}_{w} S_{0}+2 \bar{\partial} \hat{\alpha} \bar{\partial} S_{-1}=0
\end{aligned}
$$

With the result (3.86) the first equation gives $\partial_{w} S_{0}=0$, while the second equation is automatically satisfied because $\bar{\partial}_{w} S_{-1}=0$. To fix $S_{0}$ completely, one needs to look at the next order of the expansion. One can then deduce an additional equation $\bar{\partial}_{w} S_{0}=0$ and hence the solution is $S_{0}=$ constant, which can be absorbed into the normalization constant.

Continuing in this fashion, we arrive at the following result for the WKB expansion of $\tilde{\psi}_{i}$, up to $\mathcal{O}(\xi)$ :

$$
\begin{aligned}
& \tilde{\psi}_{1}=\exp \left[ \pm\left(\frac{1}{\xi} \int d w+\xi\left(\frac{1}{2} \int d w\left\{\left(\partial_{w} \hat{\alpha}\right)^{2}-\partial_{w}^{2} \hat{\alpha}\right\}+\int d \bar{w} e^{-2 \hat{\alpha}}\right)+\cdots\right)\right] \\
& \tilde{\psi}_{2}= \pm \exp \left[ \pm\left(\frac{1}{\xi} \int d w+\xi\left(\partial_{w} \hat{\alpha}\left(w_{i}\right)+\frac{1}{2} \int d w\left\{\left(\partial_{w} \hat{\alpha}\right)^{2}+\partial_{w}^{2} \hat{\alpha}\right\}+\int d \bar{w} e^{2 \hat{\alpha}}\right)+\cdots\right)\right] .
\end{aligned}
$$


It turns out that it is more convenient to reorganize this result by grouping the terms in the exponent of $\tilde{\psi}_{1}$ into two parts which are even or odd under $\xi \rightarrow-\xi$. Namely we write (3.84) as

$$
\tilde{\psi}_{1}=\exp \left[S_{\text {odd }}+S_{\text {even }}\right]
$$

Substituting this into the equations for $\tilde{\psi}_{1}$, namely (3.80) and (3.81), we can deduce four separate equations relating $S_{\text {even }}$ and $S_{\text {odd }}$. One can actually obtain two more equations from (3.82) and (3.83), expressing the relations between $\tilde{\psi}_{1}$ and $\tilde{\psi}_{2}$. Combining these set of equations, one can deduce, in particular, the following simple equation expressing $S_{\text {even }}$ in terms of $S_{\text {odd }}$ :

$$
S_{\text {even }}=-\frac{1}{2} \ln \partial_{w} S_{\text {odd }}
$$

Using this relation, we can express $\tilde{\psi}_{i}$ compactly as

$$
\begin{aligned}
& \tilde{\psi}_{1}=\frac{1}{\sqrt{\xi \partial_{w} S_{o d d}}} \exp \left[ \pm S_{\text {odd }}^{+}\right] \\
& \tilde{\psi}_{2}= \pm \sqrt{\xi \partial_{w} S_{o d d}^{+}}\left(1 \pm \frac{\bar{\partial}_{w} S_{\text {even }}}{\bar{\partial}_{w} S_{\text {odd }}^{+}}\right) \exp \left[ \pm S_{\text {odd }}^{+}\right] .
\end{aligned}
$$

Here the symbol $S_{\text {odd }}^{+}$signifies the value of $S_{\text {odd }}$ when the sign in the solution for $S_{-1}$ is plus, namely for $S_{-1}=+w$ case. More explicitly, switching back to the coordinate $z$ in the expression (3.89), $S_{\text {odd }}^{+}$is given by

$$
S_{\text {odd }}^{+}=\frac{1}{\xi} \int_{z^{*}} d z \sqrt{p}+\xi\left(\frac{1}{2} \int_{z^{*}} d z\left\{\frac{1}{\sqrt{p}}(\partial \hat{\alpha})^{2}-\partial\left(\frac{1}{\sqrt{p}} \partial \hat{\alpha}\right)\right\}+\int_{z^{*}} d \bar{z} \sqrt{\bar{p}} e^{-2 \hat{\alpha}}\right)+\cdots
$$

The initial point of the integration $z^{*}$ can be chosen arbitrarily. Since a different choice only gives a shift by a constant, derivatives of $S_{\text {odd }}^{+}$are independent of $z^{*}$.

\subsubsection{Small and big solutions and $\mathrm{SL}(2, \mathrm{C})$ invariant products of small solu- tions}

We now focus on the behavior the WKB solutions near the singularities at $z_{i}$. At a generic value of $\xi$, one of the solutions in (3.93) increases while the other decreases as one approaches $z_{i}$. The former is called the big solution $b_{i}$ and the latter the small solution $s_{i}$. This is controlled by the behavior of the leading term in the exponent and hence the sign in front of $S_{\text {odd }}^{+}$.

Which sign corresponds to the small solution depends on two factors. One is the position of the singularity $z_{i}$. A solution with a particular sign which behaves as $s_{i}$ at $z_{i}$ 
may behave as a big solution $b_{j}$ at $z_{j}$. In fact if $z_{i}$ and $z_{j}$ are connected by a WKB curve, such a situation must occur. In that case, the sign of the exponent of the solution which is identified as $s_{j}$ at $z_{j}$ is opposite to the one for $s_{i}$. Another factor is the phase of $\xi$. For example, the big and small solutions are interchanged under $\xi \rightarrow e^{i \pi} \xi$.

Let us clarify the relation between the solutions $i_{ \pm}$, introduced in the previous subsection, and the small and big solutions $s_{i}$ and $b_{i}$ discussed here. $s_{i}$ must coincide (up to normalization) with one of $i_{ \pm}$since such a decreasing solution is uniquely specified with a definite asymptotic behavior. On the other hand $b_{i}$ is ambiguous since it can contain a multiple of $s_{i}$. Thus the correspondence is $\left\{i_{ \pm}, i_{\mp}\right\} \Leftrightarrow\left\{s_{i}, b_{i}+\alpha_{i} s_{i}\right\}$. It will be of utmost importance to understand which of $i_{ \pm}$is $s_{i}$ at each $z_{i}$. This is dictated by the analyticity of the solutions on the double cover $D$ of the worldsheet. Also it is intertwined with the analyticity in the $\xi$-plane as it depends on the phase of $\xi$.

Now for the problem at hand, reconstructing the original solution from (3.93) by the gauge transformation (3.78), we can define the small solution $s_{i}$, with normalization fixed, by

$$
s_{i}=\frac{e^{-i \pi / 4}}{\sqrt{2}}\left(\begin{array}{c}
e^{\alpha / 2}\left( \pm \xi \partial S_{(i), \text { odd }}^{+}\right)^{-1 / 2} \\
e^{-\alpha / 2}\left( \pm \xi \partial S_{(i), \text { odd }}^{+}\right)^{1 / 2}\left(1 \pm \frac{\bar{\partial} S_{(i), \text { even }}}{\bar{\partial} S_{(i), \text { odd }}^{+}}\right)
\end{array}\right) \exp \left[ \pm S_{(i), \text { odd }}^{+}\right]
$$

Here, $S_{(i), \text { odd }}^{+}$denotes $S_{\text {odd }}^{+}$in which the initial point $z^{*}$ of the integrals is chosen at the special point $z_{i}^{*}$ defined in subsection 3.3. Of course it is understood that the choice of the sign of the exponent is made such that $s_{i}$ decrease as $z$ approaches $z_{i}$. Once we define $s_{i}$ this way, the big solution $b_{i}$, which satisfies the normalization condition $\left\langle s_{i}, b_{i}\right\rangle=1$, can be given, with the opposite sign choice, by

$$
b_{i}=\frac{ \pm e^{-i \pi / 4}}{\sqrt{2}}\left(\begin{array}{c}
e^{\alpha / 2}\left(\mp \xi \partial S_{(i), \text { odd }}^{+}\right)^{-1 / 2} \\
e^{-\alpha / 2}\left(\mp \xi \partial S_{(i), \text { odd }}^{+}\right)^{1 / 2}\left(1 \mp \frac{\bar{\partial} S_{(i), \text { even }}}{\bar{\partial} S_{(i), \text { odd }}^{(}}\right)
\end{array}\right) \exp \left[\mp S_{(i), \text { odd }}^{+}\right] .
$$

Ambiguity of $b_{i}$ of the form of $\alpha_{i} s_{i}$ does not affect the normalization condition since $\left\langle s_{i}, s_{i}\right\rangle=0$.

Let us now derive the form of the WKB expansion of $\left\langle s_{i}, s_{j}\right\rangle$, where $z_{i}$ and $z_{j}$ are connected by a WKB curve. Such products will play crucial roles in the computation of the regularized area in subsection 3.6.

What is important here is that the signs of the exponent of such WKB-connected small solutions $s_{i}$ and $s_{j}$ are opposite. Suppose the small solution $s_{i}$ corresponds to the plus sign, i.e. $s_{i}=i_{+}$in the notation of subsection 3.4. Then that solution when followed 
to the point $z_{j}$ along the WKB curve must be identified as the big solution $b_{j}$ there. This means that the small solution $s_{j}$, which is the other independent solution at $z_{j}$, must have the sign opposite to that of $b_{j}$ and is identified as $s_{j}=j_{-}$. Similarly, if $s_{i}=i_{-}$then $s_{j}=j_{+}$. Therefore we can write the WKB expansion of $s_{i}$ and $s_{j}$ as

$$
\begin{aligned}
& s_{i}=\frac{e^{-i \pi / 4}}{\sqrt{2}}\left(\begin{array}{c}
e^{\alpha / 2}\left( \pm \xi \partial S_{(i) \text { odd }}^{+}\right)^{-1 / 2} \\
e^{-\alpha / 2}\left( \pm \xi \partial S_{(i), \text { odd }}^{+}\right)^{1 / 2}\left(1 \pm \frac{\bar{\partial} S_{(i), \text { even }}}{\bar{\partial} S_{(i), \text { odd }}^{+}}\right)
\end{array}\right) \exp \left[ \pm S_{(i), \text { odd }}^{+}\right] \\
& s_{j}=\frac{e^{-i \pi / 4}}{\sqrt{2}}\left(\begin{array}{c}
e^{\alpha / 2}\left(\mp \xi \partial S_{(j), \text { odd }}^{+}\right)^{-1 / 2} \\
e^{-\alpha / 2}\left(\mp \xi \partial S_{(j), \text { odd }}^{+}\right)^{1 / 2}\left(1 \mp \frac{\bar{\partial} S_{(j), \text { even }}}{\bar{\partial} S_{(j), \text { odd }}^{+}}\right)
\end{array}\right) \exp \left[\mp S_{(j), \text { odd }}^{+}\right] .
\end{aligned}
$$

Note that the prefactors in front of the exponential parts in these expressions depend only on the derivatives of $S_{\text {odd }}^{+}$, which as remarked previously do not depend on the initial points $z_{i}^{*}$ of the line integrals and hence on the subscripts $(i)$ and $(j)$. For this reason, in the product $\left\langle s_{i}, s_{j}\right\rangle$ these prefactors cancel exactly and the result takes the following simple form:

$$
\left\langle s_{i}, s_{j}\right\rangle= \pm \exp \left[ \pm S_{i \rightarrow j}\right]
$$

Here the symbol $S_{i \rightarrow j}$ is defined by

$$
\begin{aligned}
S_{i \rightarrow j} & =S_{(i), \text { odd }}^{+}-S_{(j), \text { odd }}^{+} \\
& =\frac{1}{\xi} \int_{z_{i}^{*}}^{z_{j}^{*}} d z \sqrt{p}+\xi\left(\frac{1}{2} \int_{z_{i}^{*}}^{z_{j}^{*}} d z\left\{\frac{1}{\sqrt{p}}(\partial \hat{\alpha})^{2}-\partial\left(\frac{1}{\sqrt{p}} \partial \hat{\alpha}\right)\right\}+\int_{z_{i}^{*}}^{z_{j}^{*}} d \bar{z} \sqrt{\bar{p}} e^{-2 \hat{\alpha}}\right)+\cdots,
\end{aligned}
$$

where the integration is performed along the WKB curve. The signs in (3.99) are defined to be in unison with those of the exponent of $s_{i}$. That is, if the exponential part of $s_{i}$ is $\exp \left[+S_{(i), \text { odd }}^{+}\right]$, then $\left\langle s_{i}, s_{j}\right\rangle=+\exp \left[+S_{i \rightarrow j}\right]$ and so on.

It is actually more convenient to express the formula (3.100) in a manner independent of the explicit positions $i, j$ of $s_{i}$ and $s_{j}$. For this purpose, we introduce a notation $s_{\oplus}$ for a small solution for which the exponent of its WKB expansion is of the form $\exp \left[+S_{\text {odd }}^{+}\right]$, without referring to the position around which it is a small solution. The symbol $s_{\ominus}$ should be understood in the same obvious manner. In other words,

$$
s_{\oplus} \sim \exp \left[\frac{1}{\xi} \int_{z_{\oplus}^{*}} d z \sqrt{p}+\cdots\right], \quad s_{\ominus} \sim \exp \left[-\frac{1}{\xi} \int_{z_{\ominus}^{*}} d z \sqrt{p}+\cdots\right] .
$$


Then, the formula (3.100) can be expressed, for any pair of points connected by a WKB curve, as

$$
\begin{aligned}
& \left\langle s_{\oplus}, s_{\ominus}\right\rangle=\exp \left[S_{\oplus \rightarrow \ominus}\right] \\
& S_{\oplus \rightarrow \ominus}=\frac{1}{\xi} \int_{z_{\oplus}^{*}}^{z_{\ominus}^{*}} d z \sqrt{p}+\xi\left(\frac{1}{2} \int_{z_{\oplus}^{*}}^{z_{\ominus}^{*}} d z\left\{\frac{1}{\sqrt{p}}(\partial \hat{\alpha})^{2}-\partial\left(\frac{1}{\sqrt{p}} \partial \hat{\alpha}\right)\right\}+\int_{z_{\oplus}^{*}}^{z_{\ominus}^{*}} d \bar{z} \sqrt{\bar{p}} e^{-2 \hat{\alpha}}\right)+\cdots
\end{aligned}
$$

Now an important observation is that a part of $S_{\oplus \rightarrow \ominus}$ above can be expressed in terms of the contour integrals $\int \lambda d z$ and $\int u d \bar{z}+v d z$, which appear in the expression of the regularized area. The integrals $\int \lambda d z$ and $\int v d z$ are visible already. To recognize the structure $\int u d \bar{z}$, we must rewrite the last term of the order $\xi$ part using the equation of motion for $\hat{\alpha}$, which reads

$$
\frac{\partial \bar{\partial} \hat{\alpha}}{\sqrt{p \bar{p}}}-e^{2 \hat{\alpha}}+e^{-2 \hat{\alpha}}=0 .
$$

Then it is easy to rewrite $S_{\oplus \rightarrow \ominus}$ into the form

$$
\begin{aligned}
S_{\oplus \rightarrow \ominus}= & \frac{1}{\xi} \int_{z_{\oplus}^{*}}^{z_{\ominus}^{*}} \lambda d z+\xi \int_{z_{\oplus}^{*}}^{z_{\ominus}^{*}} \sqrt{\bar{p}} d \bar{z} \\
& +\frac{\xi}{2}\left(\int_{z_{\oplus}^{*}}^{z_{\ominus}^{*}}(u d \bar{z}+v d z)-\int_{z_{\oplus}^{*}}^{z_{\ominus}^{*}} \partial\left(\frac{1}{\sqrt{p}} \partial \hat{\alpha}\right) d z+\bar{\partial}\left(\frac{1}{\sqrt{p}} \partial \hat{\alpha}\right) d \bar{z}\right)+\cdots
\end{aligned}
$$

Notice that the last term is an integral over a total derivative of $\partial \hat{\alpha} / \sqrt{p}$. In the case of LSGKP strings, this contribution vanishes since $\partial \hat{\alpha} \rightarrow 0$ at each singularity. Therefore for our problem $S_{\oplus \rightarrow \ominus}$ simplifies to

$$
S_{\oplus \rightarrow \ominus}=\frac{1}{\xi} \int_{z_{\oplus}^{*}}^{z_{\ominus}^{*}} \lambda d z+\xi \int_{z_{\oplus}^{*}}^{z_{\ominus}^{*}} \sqrt{\bar{p}} d \bar{z}+\frac{\xi}{2} \int_{z_{\oplus}^{*}}^{z_{\ominus}^{*}}(u d \bar{z}+v d z)+\cdots .
$$

So far we have been considering the WKB expansion around $\xi=0$. The expansion around $\xi=\infty$ can be carried out in a similar way. In particular, for that expansion the leading term around $\xi=\infty$ is given by

$$
S_{\oplus \rightarrow \ominus}=\xi \int_{z_{\oplus}^{*}}^{z_{\ominus}^{*}} \sqrt{\bar{p}} d \bar{z}+\cdots
$$

These results will be used to determine the analyticity of $S L(2, C)$ invariant products in section 3.6, which in turn leads to their evaluation. 


\subsection{Regularized area from $\mathrm{SL}(2, \mathrm{C})$-invariant products}

We are now finally in a position to combine all the results of the preceding subsections and derive the formula for the regularized area in terms of the parameters $\kappa_{i}$ which characterize GKP strings. The remaining steps are as follows. First, we express the contour integrals given in (3.41) in terms of the $S L(2, C)$ invariant products between small solutions, duly distinguishing the different domains of the value of $\xi$. We then clarify the precise relations between the small solutions and the plus-minus solutions. Together with the result obtained in subsection 3.4, this will allow us to express the contour integrals expressing the regularized area in terms of $\kappa_{i}$ in a completely explicit manner. The final result will be the formula (3.142).

\subsubsection{WKB curves for a specific parameter regime}

As we saw in subsections 3.3 and 3.5, both the regularized area of our interest and the WKB expansion of the invariant products $\left\langle s_{i}, s_{j}\right\rangle$ of small solutions can be expressed in terms of the same type of integrals, namely $\int \lambda d z$ and $\int(u d \bar{z}+v d z)$. To make use of this result, however, the integration contours in both cases must be related. Since the WKB expansion can only be performed reliably along WKB curves, this means that we must obtain the precise knowledge of the WKB curves and express the contours relevant to the regularized area in terms of such curves.

As the structure of the WKB curves strongly depend on the phase of $\xi$ and on the residues $\delta_{i}$ of the poles, it is not useful to try to discuss the general situation from the beginning. In the case of the GKP strings, $\delta_{i}$ 's are pure imaginary and related to $\kappa_{i}$ as

$$
\delta_{i}=\frac{i \kappa}{2} .
$$

Therefore we shall first focus on a specific case where the parameters are in the range

$$
\begin{aligned}
& \operatorname{Im} \xi>0, \\
& \kappa_{2}>\kappa_{1}, \quad \kappa_{2}>\kappa_{3}, \quad \kappa_{1}+\kappa_{3}>\kappa_{2} .
\end{aligned}
$$

The case for $\operatorname{Im} \xi<0$ will be considered later in subsection 3.6.4 and the cases with more general configurations of $\kappa_{i}$ will be treated in subsection 3.6.5 and the appendix A. Typical configurations of WKB curves are shown in figure 3.10, which are obtained by using the formula (3.23). The important feature of the WKB curves in this parameter regime is that every pair of poles are connected directly by a WKB curve and hence the contours $d_{j}$, which were defined at the end of subsection 3.3 and will play crucial roles, can easily be expressed in terms of three such WKB curves. (As we shall see later, this is not the case for generic $\kappa_{i}$.) 


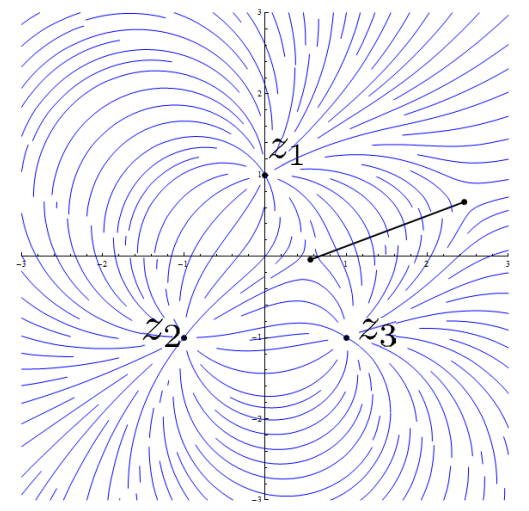

Figure 3.10: Typical configuration of WKB curves for the parameter regime where the triangle inequality holds. The parameters used are $\kappa_{1}=\kappa_{3}=1, \kappa_{2}=\sqrt{3}$ and $\operatorname{Im} \xi<0$.

\subsubsection{Relation between contour integrals and the products $\left\langle s_{i}, s_{j}\right\rangle$}

Having seen the structure of the WKB curves in the parameter regime defined in (3.106) and (3.107), we now relate the contour integrals along $d_{i}$ 's and the products $\left\langle s_{i}, s_{j}\right\rangle$ explicitly. To do this, we must first specify the branch structure of the function $\sqrt{p(z)}$. We will take the square root branch cut to be along a line which connects the two zero's of $p(z)$. Then the behavior of $\sqrt{p(z)}$ on the double cover of the worldsheet is automatically dictated once we specify the behavior around one of its poles. In what follows, we do this by setting the behavior around $z_{1}$ on the first sheet to be $\sqrt{p(z)} \stackrel{z \sim z_{1}}{\sim} \delta_{1} /\left(z-z_{1}\right)$. This determines the behaviors on the first sheet around the poles to be

$$
\begin{aligned}
\sqrt{p(z)} & \stackrel{z \sim z_{1}}{\sim} \frac{\delta_{1}}{z-z_{1}}=\frac{i \kappa_{1}}{2\left(z-z_{1}\right)}, \\
z \sim z_{2} \frac{-\delta_{2}}{z-z_{2}} & =\frac{-i \kappa_{2}}{2\left(z-z_{2}\right)}, \\
& z \sim z_{3} \frac{\delta_{3}}{z-z_{3}}=\frac{i \kappa_{3}}{2\left(z-z_{3}\right)} .
\end{aligned}
$$

The corresponding behaviors on the second sheet are obtained by flipping the overall sign. With this definition, the eigenvalues of the monodromy matrices are given by

$$
\rho_{1}=2 \pi \delta_{1}\left(\frac{1}{\xi}+\xi\right), \quad \rho_{2}=-2 \pi \delta_{2}\left(\frac{1}{\xi}+\xi\right), \quad \rho_{3}=2 \pi \delta_{3}\left(\frac{1}{\xi}+\xi\right)
$$

and the small solutions and the plus-minus solutions are related as

$$
s_{1} \sim 1_{+}, \quad s_{2} \sim 2_{-}, \quad s_{3} \sim 3_{+}
$$

Here $\sim$ symbol means that the relation is yet up to normalization. 
With this preparation we can now apply (3.101) to relate the products $\left\langle s_{i}, s_{j}\right\rangle$ with the contour integrals on $d_{i}$. We will illustrate this by taking the contour $d_{2}$ as an example. From the definition of $d_{2}$, it can be decomposed into three WKB curves as

$$
d_{2}=\left(\widehat{z}_{2} \rightarrow \widehat{z}_{3}\right)+\left(\widehat{z}_{3} \rightarrow z_{1}\right)+\left(z_{1} \rightarrow z_{2}\right),
$$

where $\left(z_{i} \rightarrow z_{j}\right)$ denotes a WKB curve connecting $z_{i}$ and $z_{j}$ and the symbol $\widehat{z}_{i}$ with a hat reminds us that the point is on the second sheet.

We first compute the contribution from the last part, $\left(z_{1} \rightarrow z_{2}\right)$ by using the formula (3.103) developed in the previous subsection. Since $s_{1}$ and $s_{2}$ are related to plus-minus solutions as in (3.109), the symbols $z_{\oplus}^{*}$ and $z_{\ominus}^{*}$ in the formula correspond to $z_{1}$ and $z_{2}$ respectively. Therefore, the logarithm of $\left\langle s_{1}, s_{2}\right\rangle$ can be evaluated as

$$
\log \left\langle s_{1}, s_{2}\right\rangle=\left(\xi^{-1} \int_{z_{1}}^{z_{2}} \sqrt{p} d z+\xi \int_{z_{1}}^{z_{2}} \sqrt{\bar{p}} d \bar{z}\right)+\frac{\xi}{2}\left(\int_{z_{1}}^{z_{2}} u d \bar{z}+v d z\right)+\cdots
$$

Similarly, for the contribution coming from the WKB curve $\left(\widehat{z}_{3} \rightarrow z_{1}\right)$, $z_{\oplus}^{*}$ corresponds to $z_{1}$, while $z_{\ominus}^{*}$ is identified as $\widehat{z}_{3}$ since the sign of $\sqrt{p(z)}$ is flipped on the second sheet. We therefore obtain

$$
\log \left\langle s_{1}, s_{3}\right\rangle=\left(\xi^{-1} \int_{z_{1}}^{\widehat{z}_{3}} \sqrt{p} d z+\xi \int_{z_{1}}^{\widehat{z}_{3}} \sqrt{\bar{p}} d \bar{z}\right)+\frac{\xi}{2}\left(\int_{z_{1}}^{\widehat{z}_{3}} u d \bar{z}+v d z\right)+\cdots
$$

Finally, in the same way, WKB expansion of $\left\langle s_{2}, s_{3}\right\rangle$ along $\left(\widehat{z}_{2} \rightarrow \widehat{z}_{3}\right)$ gives the formula

$$
\log \left\langle s_{2}, s_{3}\right\rangle=\left(\xi^{-1} \int_{\widehat{z}_{2}}^{\widehat{z}_{3}} \sqrt{p} d z+\xi \int_{\widehat{z}_{2}}^{\widehat{z}_{3}} \sqrt{\bar{p}} d \bar{z}\right)+\frac{\xi}{2}\left(\int_{\widehat{z}_{2}}^{\widehat{z}_{3}} u d \bar{z}+v d z\right)+\cdots
$$

Putting the three contributions together, we get the expression for the integrals along $d_{2}$, which is displayed below together with the ones along $d_{1}$ and $d_{3}$ obtained by similar analysis:

$$
\begin{aligned}
& \frac{\left\langle s_{2}, s_{3}\right\rangle}{\left\langle s_{2}, s_{1}\right\rangle\left\langle s_{1}, s_{3}\right\rangle}=\exp \left[\frac{1}{\xi} \int_{d_{1}} \lambda d z+\xi \int_{d_{1}} \sqrt{\bar{p}} d \bar{z}+\frac{\xi}{2}\left(\int_{d_{1}} u d \bar{z}+v d z\right)+\cdots\right], \\
& \frac{\left\langle s_{2}, s_{3}\right\rangle\left\langle s_{1}, s_{2}\right\rangle}{\left\langle s_{1}, s_{3}\right\rangle}=\exp \left[\frac{1}{\xi} \int_{d_{2}} \lambda d z+\xi \int_{d_{2}} \sqrt{\bar{p}} d \bar{z}+\frac{\xi}{2}\left(\int_{d_{2}} u d \bar{z}+v d z\right)+\cdots\right], \\
& \frac{\left\langle s_{1}, s_{2}\right\rangle}{\left\langle s_{1}, s_{3}\right\rangle\left\langle s_{3}, s_{2}\right\rangle}=\exp \left[\frac{1}{\xi} \int_{d_{3}} \lambda d z+\xi \int_{d_{3}} \sqrt{\bar{p}} d \bar{z}+\frac{\xi}{2}\left(\int_{d_{3}} u d \bar{z}+v d z\right)+\cdots\right] .
\end{aligned}
$$

As we already calculated, in subsection 3.3, the $S L(2, C)$ products between plusminus solutions in terms of the local monodromy data (3.108), if we can identify $s_{i}$ with $i_{ \pm}$precisely, the contour integrals can be expressed in terms of the parameters $\delta_{i}$. This however is not straightforward. The reason is that since the plus-minus solutions are 
defined only up to $\xi$-dependent scaling factor, the relations such as (3.109) suffer from that ambiguity. Such uncontrolled $\xi$ dependence ruins the identification of the coefficient of each power of $\xi$. Therefore it is extremely important to understand the conditions under which we can identify $s_{i}=i_{+}$(or $i_{-}$) precisely, without $\xi$-dependent relative factors.

This turned out to be intimately connected with the analyticity in $\xi$. To show this, as a first step, we study how the computation of the contour integrals described above changes as we vary $\xi$ to the region where $\operatorname{Im} \xi$ is negative. The crucial change is that the relation between the small solutions and the plus-minus solutions gets altered like

$$
\begin{array}{ll}
\operatorname{Im} \xi>0: & s_{1} \sim 1_{+}, s_{2} \sim 2_{-}, s_{3} \sim 3_{+} \\
\operatorname{Im} \xi<0: & s_{1} \sim 1_{-}, s_{2} \sim 2_{+}, \quad s_{3} \sim 3_{-} .
\end{array}
$$

This change inevitably affects the WKB expansion of the $S L(2, C)$ invariant products: The poles which were previously of the type $z_{\oplus}$ are now changed to $z_{\ominus}$ and vice versa. This modifies the relation between the contour integrals on $d_{i}$ and the $S L(2, C)$ invariant products to

$$
\begin{aligned}
& \frac{\left\langle s_{1}, s_{2}\right\rangle\left\langle s_{3}, s_{1}\right\rangle}{\left\langle s_{3}, s_{2}\right\rangle}=\exp \left[\frac{1}{\xi} \int_{d_{1}} \lambda d z+\xi \int_{d_{1}} \sqrt{\bar{p}} d \bar{z}+\frac{\xi}{2}\left(\int_{d_{1}} u d \bar{z}+v d z\right)+\cdots\right], \\
& \frac{\left\langle s_{3}, s_{1}\right\rangle}{\left\langle s_{3}, s_{2}\right\rangle\left\langle s_{2}, s_{1}\right\rangle}=\exp \left[\frac{1}{\xi} \int_{d_{2}} \lambda d z+\xi \int_{d_{2}} \sqrt{\bar{p}} d \bar{z}+\frac{\xi}{2}\left(\int_{d_{2}} u d \bar{z}+v d z\right)+\cdots\right], \\
& \frac{\left\langle s_{3}, s_{1}\right\rangle\left\langle s_{2}, s_{3}\right\rangle}{\left\langle s_{2}, s_{1}\right\rangle}=\exp \left[\frac{1}{\xi} \int_{d_{3}} \lambda d z+\xi \int_{d_{3}} \sqrt{\bar{p}} d \bar{z}+\frac{\xi}{2}\left(\int_{d_{3}} u d \bar{z}+v d z\right)+\cdots\right] .
\end{aligned}
$$

Compared to (3.115), the expressions on the left hand side are inverted and acquired an overall minus sign, because $\left\langle s_{i}, s_{j}\right\rangle$ gets changed into $\left\langle s_{j}, s_{i}\right\rangle=-\left\langle s_{i}, s_{j}\right\rangle$.

\subsubsection{Connection between normalization and analyticity}

Now we shall show that convenient normalization of the eigenvectors $i_{ \pm}$can be specified by imposing certain analyticity property on the $S L(2, C)$ invariant products $\left\langle i_{+}, j_{-}\right\rangle$.

Let us illustrate the idea by using a concrete example involving $\left\langle 1_{+}, 2_{-}\right\rangle$and $\left\langle 1_{-}, 2_{+}\right\rangle$. In subsection 3.4, we showed that the products $\left\langle i_{+}, j_{-}\right\rangle$can be expressed in terms of the local monodromy data $\rho_{i}$ and that their judicious combinations are free of rescaling ambiguities. We gave such an example in (3.75), which we display here again:

$$
\left\langle 1_{+}, 2_{-}\right\rangle\left\langle 2_{+}, 1_{-}\right\rangle=\frac{1}{2 \sin \rho_{1} \sin \rho_{2}}\left(\cos \left(\rho_{1}-\rho_{2}\right)-\cos \rho_{3}\right) .
$$

We wish to solve this equation for the individual product, $\left\langle 1_{+}, 2_{-}\right\rangle$and $\left\langle 2_{+}, 1_{-}\right\rangle$. We know from the preceding discussion that if $\operatorname{Im} \xi>0$, the eigenvectors $1_{+}$and $2_{-}$are proportional 
to $s_{1}$ and $s_{2}$ respectively and hence $\left\langle 1_{+}, 2_{-}\right\rangle$should be proportional to $\left\langle s_{1}, s_{2}\right\rangle$, for which we can make WKB expansion. Similarly, for $\operatorname{Im} \xi<0$, the product $\left\langle 2_{+}, 1_{-}\right\rangle$should be proportional to $\left\langle s_{2}, s_{1}\right\rangle$. Now we show that such solutions to the equation 3.121 are unique up to $\xi$-independent constant if we impose the following analyticity conditions, inspired by the WKB expansion of $\left\langle s_{1}, s_{2}\right\rangle$, in respective domain of $\xi$ :

$$
\begin{aligned}
& \log \left\langle 1_{+}, 2_{-}\right\rangle=\left(\frac{1}{\xi} \int_{z_{1}}^{z_{2}} \sqrt{p} d z+\xi \int_{z_{1}}^{z_{2}} \sqrt{\bar{p}} d \bar{z}\right)+\text { functions regular on } \operatorname{Im} \xi \geq 0, \\
& \log \left\langle 2_{+}, 1_{-}\right\rangle=\left(\frac{1}{\xi} \int_{z_{2}}^{z_{1}} \sqrt{p} d z+\xi \int_{z_{2}}^{z_{1}} \sqrt{\bar{p}} d \bar{z}\right)+\text { functions regular on } \operatorname{Im} \xi \leq 0 .
\end{aligned}
$$

To prove it, assume that there are two different solutions to (3.121) like

$$
\begin{aligned}
& \text { (i) } \quad\left\langle 1_{-}, 2_{+}\right\rangle=f, \quad\left\langle 2_{-}, 1_{+}\right\rangle=g, \\
& \text { (ii) }\left\langle 1_{-}, 2_{+}\right\rangle=f^{\prime}, \quad\left\langle 2_{-}, 1_{+}\right\rangle=g^{\prime} .
\end{aligned}
$$

Then, since $f g=f^{\prime} g^{\prime}$, we have

$$
\log f-\log f^{\prime}=\log g^{\prime}-\log g
$$

From the analyticity we imposed, the left hand side of this equation is regular on $\operatorname{Im} \xi \leq 0$, while the right hand side is regular on $\operatorname{Im} \xi \geq 0$. Therefore, both sides must be regular on the whole complex plane (including the infinity) and hence is a $\xi$-independent constant. This proves the assertion.

On the other hand, as should already be clear from the presentation above, the analyticity properties we imposed are precisely those of the WKB expansions of $\left\langle s_{1}, s_{2}\right\rangle$ if we identify small solutions and plus-minus solutions as ${ }^{12}$

$$
\begin{aligned}
& \operatorname{Im} \xi>0 \Rightarrow s_{1}=1_{+}, s_{2}=2_{-}, \\
& \operatorname{Im} \xi<0 \Rightarrow s_{1}=1_{-}, s_{2}=2_{+} .
\end{aligned}
$$

Therefore, the imposition of the analyticity is totally equivalent to fixing the normalization of $1_{ \pm}$and $2_{ \pm}$as in (3.125) and (3.126).

Summarizing, we have found that to evaluate the contour integrals we should (i) find the unique solution to (3.121) which satisfy the analyticity properties (3.122) and (3.123), and (ii) WKB-expand $\left\langle 1_{+}, 2_{-}\right\rangle$for $\operatorname{Im} \xi \geq 0$, or equivalently, $\left\langle 1_{-}, 2_{+}\right\rangle$for $\operatorname{Im} \xi \leq 0$. In what follows, we will show how these procedures can be carried out in practice to obtain the expression of the regularized area.

\footnotetext{
${ }^{12}$ Strictly speaking, the notion of small solution does not exist for $\operatorname{Im} \xi=0$. However, since the limit $\operatorname{Im} \xi \rightarrow 0$ produces no singularity, we can extend the condition of the analyticity to include $\operatorname{Im} \xi=0$ in (3.122) and (3.123).
} 


\subsubsection{Derivation of the formula for $\mathrm{SL}(2, \mathrm{C})$ products and computation of the regularized area}

As the first step, we wish to solve the equation of the type (3.121) under the prescribed analyticity. For this purpose, it should be noted that although individually $\left\langle 1_{-}, 2_{+}\right\rangle$and $\left\langle 2_{-}, 1_{+}\right\rangle$contain log-divergent terms of the form $\pm\left(\xi^{-1} \int_{z_{1}}^{z_{2}} \sqrt{p} d z+\xi \int_{z_{1}}^{z_{2}} \sqrt{\bar{p}} d \bar{z}\right)$, they cancel each other in the product. Therefore, we can only focus on the non-singular part of these $S L(2, C)$ invariant products.

Now to solve the equation (3.121) under the analyticity requirement, the following identity is useful:

$$
\frac{1}{2 \pi i} \int_{-\infty}^{\infty} d \xi^{\prime} \frac{1}{\xi^{\prime}-\xi}\left(F\left(\xi^{\prime}\right)+G\left(\xi^{\prime}\right)\right)= \begin{cases}F(\xi), & (\operatorname{Im} \xi>0) \\ -G(\xi), & (\operatorname{Im} \xi<0)\end{cases}
$$

Here, $F(\xi)$ is a function regular on the upper half plane and exponentially suppressed at large $|\xi|$, while $G(\xi)$ is function regular on the lower half plane and exponentially suppressed at large $|\xi|$. This formula can be proved using the residue theorem by closing the contour on the upper half or lower half plane. With the use of this identity, one can show that the following expressions give the nonsingular part of $\log \left\langle 2_{-}, 1_{+}\right\rangle$and $\log \left\langle 1_{-}, 2_{+}\right\rangle$:

$$
\begin{aligned}
& \log \left\langle 2_{-}, 1_{+}\right\rangle_{n . s .}=\frac{1}{2 \pi i} \int_{-\infty}^{\infty} d \xi^{\prime} \frac{1}{\xi^{\prime}-\xi} \log \left(\frac{\cos \left(\rho_{1}-\rho_{2}\right)-\cos \rho_{3}}{2 \sin \rho_{1} \sin \rho_{2}}\left(\xi^{\prime}\right)\right), \quad(\operatorname{Im} \xi>0), \\
& \log \left\langle 1_{-}, 2_{+}\right\rangle_{n . s .}=-\frac{1}{2 \pi i} \int_{-\infty}^{\infty} d \xi^{\prime} \frac{1}{\xi^{\prime}-\xi} \log \left(\frac{\cos \left(\rho_{1}-\rho_{2}\right)-\cos \rho_{3}}{2 \sin \rho_{1} \sin \rho_{2}}\left(\xi^{\prime}\right)\right), \quad(\operatorname{Im} \xi<0),
\end{aligned}
$$

where the subscript n.s. denotes the non-singular part. The expressions of these quantities on the other half of the $\xi$-plane are defined by the analytic continuation of the above results. Now rewrite the factor $\frac{1}{2}\left(\cos \left(\rho_{1}-\rho_{2}\right)-\cos \rho_{3}\right)$ as

$$
\frac{\cos \left(\rho_{1}-\rho_{2}\right)-\cos \rho_{3}}{2}=\sin \frac{\rho_{1}-\rho_{2}+\rho_{3}}{2} \sin \frac{-\rho_{1}+\rho_{2}+\rho_{3}}{2} .
$$

and substitute the forms of $\rho_{i}$ given in (3.108). Then, switching to the notation in terms of small solutions, the above expressions can be reorganized as

$$
\begin{aligned}
& \operatorname{Im} \xi>0: \\
& \log \left\langle s_{1}, s_{2}\right\rangle_{n . s .}=h\left(\kappa_{1}\right)+h\left(\kappa_{2}\right)-h\left(\frac{\kappa_{1}+\kappa_{2}+\kappa_{3}}{2}\right)-h\left(\frac{\kappa_{1}+\kappa_{2}-\kappa_{3}}{2}\right), \\
& \operatorname{Im} \xi<0: \\
& \log \left\langle s_{1}, s_{2}\right\rangle_{n . s .}=-h\left(\kappa_{1}\right)-h\left(\kappa_{2}\right)+h\left(\frac{\kappa_{1}+\kappa_{2}+\kappa_{3}}{2}\right)+h\left(\frac{\kappa_{1}+\kappa_{2}-\kappa_{3}}{2}\right),
\end{aligned}
$$


where the function $h(x)$ is defined by

$$
h(x) \equiv-\frac{1}{\pi i} \int_{0}^{+\infty} d \xi^{\prime} \frac{\xi}{\xi^{\prime 2}-\xi^{2}} \log \left(1-\exp \left(-2 \pi x\left(\xi^{\prime^{-1}}+\xi^{\prime}\right)\right)\right) .
$$

In the same manner, $\left\langle s_{2}, s_{3}\right\rangle$ and $\left\langle s_{3}, s_{1}\right\rangle$ can be expressed as

$$
\begin{aligned}
& \operatorname{Im} \xi>0: \\
& \log \left\langle s_{2}, s_{3}\right\rangle_{n . s .}=h\left(\kappa_{2}\right)+h\left(\kappa_{3}\right)-h\left(\frac{\kappa_{1}+\kappa_{2}+\kappa_{3}}{2}\right)-h\left(\frac{-\kappa_{1}+\kappa_{2}+\kappa_{3}}{2}\right), \\
& \log \left\langle s_{3}, s_{1}\right\rangle_{n . s .}=h\left(\kappa_{3}\right)+h\left(\kappa_{1}\right)-h\left(\frac{\kappa_{1}+\kappa_{2}+\kappa_{3}}{2}\right)-h\left(\frac{\kappa_{1}-\kappa_{2}+\kappa_{3}}{2}\right), \\
& \operatorname{Im} \xi<0: \\
& \log \left\langle s_{2}, s_{3}\right\rangle_{n . s .}=-h\left(\kappa_{2}\right)-h\left(\kappa_{3}\right)+h\left(\frac{\kappa_{1}+\kappa_{2}+\kappa_{3}}{2}\right)+h\left(\frac{-\kappa_{1}+\kappa_{2}+\kappa_{3}}{2}\right), \\
& \log \left\langle s_{3}, s_{1}\right\rangle_{n . s .}=-h\left(\kappa_{3}\right)-h\left(\kappa_{1}\right)+h\left(\frac{\kappa_{1}+\kappa_{2}+\kappa_{3}}{2}\right)+h\left(\frac{\kappa_{1}-\kappa_{2}+\kappa_{3}}{2} .\right.
\end{aligned}
$$

We can now finally evaluate the contour integrals of our interest. First the integrals $\int_{d_{i}}(u d \bar{z}+v d z)$ can be extracted as the coefficients of $\xi$ in the non-singular part of the expansions given in (3.115) and (3.119). Hence, we need the order $\xi$ part of the function $h(x)$. It is given by

$$
\begin{aligned}
h(x) & =i \xi K(x)+\mathcal{O}\left(\xi^{2}\right), \\
K(x) & =\frac{1}{\pi} \int_{-\infty}^{\infty} d \theta e^{-\theta} \log \left(1-e^{-4 \pi x \cosh \theta}\right) .
\end{aligned}
$$

Using this function $K(x)$, we obtain

$$
\begin{aligned}
\int_{d_{1}}(u d \bar{z}+v d z) & =2 i\left(K\left(\frac{\kappa_{1}+\kappa_{2}+\kappa_{3}}{2}\right)-2 K\left(\kappa_{1}\right)\right. \\
& \left.-K\left(\frac{-\kappa_{1}+\kappa_{2}+\kappa_{3}}{2}\right)+K\left(\frac{\kappa_{1}-\kappa_{2}+\kappa_{3}}{2}\right)+K\left(\frac{\kappa_{1}+\kappa_{2}-\kappa_{3}}{2}\right)\right) \\
\int_{d_{2}}(u d \bar{z}+v d z) & =-2 i\left(K\left(\frac{\kappa_{1}+\kappa_{2}+\kappa_{3}}{2}\right)-2 K\left(\kappa_{2}\right)\right. \\
& \left.+K\left(\frac{-\kappa_{1}+\kappa_{2}+\kappa_{3}}{2}\right)-K\left(\frac{\kappa_{1}-\kappa_{2}+\kappa_{3}}{2}\right)+K\left(\frac{\kappa_{1}+\kappa_{2}-\kappa_{3}}{2}\right)\right) \\
\int_{d_{3}}(u d \bar{z}+v d z) & =2 i\left(K\left(\frac{\kappa_{1}+\kappa_{2}+\kappa_{3}}{2}\right)-2 K\left(\kappa_{3}\right)\right. \\
& \left.+K\left(\frac{-\kappa_{1}+\kappa_{2}+\kappa_{3}}{2}\right)+K\left(\frac{\kappa_{1}-\kappa_{2}+\kappa_{3}}{2}\right)-K\left(\frac{\kappa_{1}+\kappa_{2}-\kappa_{3}}{2}\right)\right) .
\end{aligned}
$$

On the other hand, the contour integrals $\int_{C_{i}} \lambda d z$ around the poles $z_{i}$ are easily evaluated as

$$
\int_{C_{1}} \lambda d z=-\pi \kappa_{1}, \quad \int_{C_{2}} \lambda d z=+\pi \kappa_{2}, \quad \int_{C_{3}} \lambda d z=-\pi \kappa_{3}
$$


Combining the above results, together with the constant contribution discussed in subsection 3.3, the regularized area in the parameter regime (3.107) is expressed in terms of $\kappa_{i}$ as

$$
\begin{aligned}
\int d^{2} z \lambda u & =\frac{\pi}{12}+\pi\left[-\kappa_{1} K\left(\kappa_{1}\right)-\kappa_{2} K\left(\kappa_{2}\right)-\kappa_{3} K\left(\kappa_{3}\right)\right. \\
& +\frac{\kappa_{1}+\kappa_{2}+\kappa_{3}}{2} K\left(\frac{\kappa_{1}+\kappa_{2}+\kappa_{3}}{2}\right)+\frac{-\kappa_{1}+\kappa_{2}+\kappa_{3}}{2} K\left(\frac{-\kappa_{1}+\kappa_{2}+\kappa_{3}}{2}\right) \\
& \left.+\frac{\kappa_{1}-\kappa_{2}+\kappa_{3}}{2} K\left(\frac{\kappa_{1}-\kappa_{2}+\kappa_{3}}{2}\right)+\frac{\kappa_{1}+\kappa_{2}-\kappa_{3}}{2} K\left(\frac{\kappa_{1}+\kappa_{2}-\kappa_{3}}{2}\right)\right] .
\end{aligned}
$$

Since the result above is symmetric under the permutation of $\kappa_{i}$, we can apply the same formula for such permuted regimes. The characteristic feature of these regimes is that the "triangle inequality" $\kappa_{i}<\kappa_{j}+\kappa_{k}(i \neq j \neq k)$ is satisfied. Therefore, to cover the entire range of parameters, we must discuss separately the cases in which the triangle inequality is not satisfied, namely when $\kappa_{i}>\kappa_{j}+\kappa_{k}(i \neq j \neq k)$. The analysis for such regimes is more involved because the configuration of the WKB curves and the position of the branch cut change in such a way that one cannot perform the WKB expansion straightforwardly. We will explain how such a situation should be handled in the appendix A. We find, after careful analyses, that signs in the formula (3.141) must be changed appropriately. The net result, however, turned out to be quite gratifying. The general formula which is valid for all the parameter regimes can be written as

$$
\begin{aligned}
A_{\text {reg }}=\int d^{2} z \lambda u & =\frac{\pi}{12}+\pi\left[-\kappa_{1} K\left(\kappa_{1}\right)-\kappa_{2} K\left(\kappa_{2}\right)-\kappa_{3} K\left(\kappa_{3}\right)\right. \\
& +\frac{\kappa_{1}+\kappa_{2}+\kappa_{3}}{2} K\left(\frac{\kappa_{1}+\kappa_{2}+\kappa_{3}}{2}\right) \\
& +\left|\frac{-\kappa_{1}+\kappa_{2}+\kappa_{3}}{2}\right| K\left(\left|\frac{-\kappa_{1}+\kappa_{2}+\kappa_{3}}{2}\right|\right) \\
& +\left|\frac{\kappa_{1}-\kappa_{2}+\kappa_{3}}{2}\right| K\left(\left|\frac{\kappa_{1}-\kappa_{2}+\kappa_{3}}{2}\right|\right) \\
& \left.+\left|\frac{\kappa_{1}+\kappa_{2}-\kappa_{3}}{2}\right| K\left(\left|\frac{\kappa_{1}+\kappa_{2}-\kappa_{3}}{2}\right|\right)\right] .
\end{aligned}
$$

This is the final result expressing the most important part of the three point coupling of the LSGKP strings, in terms of the parameters $\kappa_{i}$ by which the AdS energy and the spin of the string are expressed. Substituting this result into (3.7), we obtain the fully explicit expression of the finite part of the area $A_{f i n}$. 


\section{Semi-classical wave function and vertex operator for three-point functions}

In the previous section, we have computed the $S L(2, C)$ invariant finite part of the area $A_{\text {fin }}$, which constitutes the main part of the three point function for the LSGKP strings. In what follows, we will discuss the remaining part of the three point function made up of the divergent part of the area defined in subsection 3.1 and the contribution of the vertex operators. We will argue that these contributions together give finite result and explain how the appropriate vertex operator should be constructed systematically. Although such a program has not yet been carried out for the LSGKP strings, we will illustrate our arguments by using an example of rotating strings in flat spacetime.

\subsection{Structure of three-point functions}

As described in the introduction, the remaining part of the area

$$
A_{d i v}=4 \int d^{2} z \sqrt{p \bar{p}}
$$

and the contribution from the vertex operators

$$
\sum_{i} \log V_{i}\left[X_{*} ; z_{i}, x_{i}, Q_{i}\right]
$$

diverge separately. Therefore, to compute these quantities properly one must introduce appropriate regularization parameters for each of the contributions. As will be illustrated later for the case of rotating string in flat space, if the regularization parameters are judiciously taken, the dependence on them will cancel each other and one obtains a finite result.

To demonstrate such a mechanism for the LSGKP strings and obtain complete three point functions, we must first construct the semi-classical vertex operators for LSGKP. In string theory, the correct vertex operator for a closed string state must satisfy two requirements:

- First it must transform correctly under the global symmetry group so that it carries the target space quantum numbers of the state in question. This requirement is relatively easy to satisfy as string coordinates which transform covariantly are usually available.

- The second requirement is that it must be a conformal primary of dimension $(1,1)$ on the worldsheet. In the present case, where we are interested in states with large 
quantum numbers, $(0,0)$ primarity is sufficient within the saddle point approximation.

One way to check that a candidate vertex operator is indeed such a primary is to evaluate its two point function, as discussed in [26], and see if the result does not depend on the insertion points on the worldsheet. This method is rather cumbersome as one needs to evaluate the entire two point function. We will make a comment on it shortly.

Below we propose an alternative simpler method, apparently not discussed in the literature, which is a saddle point version of the conformal Ward identity.

As is well-known, the correlation functions of the primary operators $V_{i}$ with dimension $\Delta_{i}$ satisfy the following Ward identity:

$$
\begin{aligned}
& \left\langle T(z) V_{1}\left(z_{1}\right) V_{2}\left(z_{2}\right) \cdots V_{n}\left(z_{n}\right)\right\rangle \\
& =\sum_{i=1}^{n}\left(\frac{\Delta_{i}}{\left(z-z_{i}\right)^{2}}+\frac{1}{z-z_{i}} \frac{\partial}{\partial z_{i}}\right)\left\langle V_{1}\left(z_{1}\right) V_{2}\left(z_{2}\right) \cdots V_{n}\left(z_{n}\right)\right\rangle .
\end{aligned}
$$

It should be emphasized that the vertex operators, as well as the energy-momentum tensor, must be properly renormalized so that the correlation functions are finite. When the theory is not free, such as in the case at hand, this renormalization procedure is in general quite non-trivial. Now when all the vertex operators carry large quantum numbers, of the order of a large number $\gamma, V_{i}$ can be expressed as

$$
V_{i}=e^{\gamma v_{i}}
$$

In such a case, since the $T(z)$ itself is an operator with small quantum number, the insertion of $T(z)$ does not affect the saddle. Therefore we can evaluate (4.3) with the same saddle configuration appropriate for the correlation function without the insertion of $T(z)$. Now since the correct saddle point configuration must satisfy the Virasoro condition, the left hand side vanishes. In particular, for the two point function the identity becomes

$$
\begin{aligned}
0 & =\frac{1}{z-z_{1}}\left\langle\partial_{1} V_{1}\left(z_{1}\right) V_{2}\left(z_{2}\right)\right\rangle+\frac{1}{z-z_{2}}\left\langle V_{1}\left(z_{1}\right) \partial_{2} V_{2}\left(z_{2}\right)\right\rangle \\
& =\left(\left.\sum_{i} \frac{\gamma}{z-z_{i}} \frac{\partial v_{i}\left(z_{i}\right)}{\partial z_{i}}\right|_{\text {saddle }}\right)\left\langle V_{1}\left(z_{1}\right) V_{2}\left(z_{2}\right)\right\rangle .
\end{aligned}
$$

Since $z$ is still arbitrary, this is equivalent to the conditions

$$
\left.\frac{\partial v_{i}\left(z_{i}\right)}{\partial z_{i}}\right|_{\text {saddle }}=0 .
$$

which must hold at each point $z_{i}$. From similar consideration with the insertion of $\bar{T}(\bar{z})$, we obtain the same relations with $\partial$ replaced by $\bar{\partial}$. This is the simple form of the conformal 
Ward identity valid for the saddle point approximation and one can use it to check if the vertex operators are conformally correct.

Here we should make a clarifying remark on the passage from (4.3) to the expression (4.5). In the path integral formulation the symbol $\langle\cdots\rangle$ contains the weight factor $e^{-S}$, where $S$ is the action. Since $S$ is independent of the coordinates $z_{i}$ of the vertex operators, it is legitimate to bring the derivatives $\partial_{i}$ inside the bracket $\langle\cdots\rangle$ as in (4.5). However, if we choose to compute the two-point function using the the saddle point method before applying the derivatives $\partial_{i}$, the action $S$ as well as the vertex operators themselves acquire extra dependence on $z_{i}$ through the saddle point cofiguration $X_{*}\left(z ; z_{1}, z_{2}\right)$, which certainly depends on the positions of the vertex operators. The derivatives $\partial_{i}$ must then act on such $z_{i}$ dependence as well. Fortunately, this apparent complication is actually absent for a simple reason. Because such extra contributions are produced only through the functional dependence on $X_{*}\left(z ; z_{1}, z_{2}\right)$, the derivative $\partial_{i}$ acts as $\int d^{2} z \partial_{i} X_{*}\left(z ; z_{1}, z_{2}\right) \delta / \delta X_{*}\left(z ; z_{1}, z_{2}\right)$. When $\delta / \delta X_{*}\left(z ; z_{1}, z_{2}\right)$ acts on the expression $\left.V_{1} V_{2} e^{-S}\right|_{\text {saddle }}$, it generates the equation of motion with $-\sum_{i} \ln V_{i}$ as the source terms. Since $X_{*}$ is precisely a solution to such an equation, this vanishes identically and again we obtain (4.5).

Now if we apply (4.7) to simple (properly renormalized) vertex operators in flat spacetime, one can check that it is equivalent, within the precision of the approximation, to the on-shell condition. On the other hand, applying it to the vertex operator of the form

$$
(\partial X \bar{\partial} X)^{S / 2}\left(X_{-1}+X_{4}\right)^{-\Delta}
$$

which is used in [26] for the LSGKP string, one finds that it is not satisfied due to the dependence on the $\sigma$ coordinate of the worldsheet. Also, it should be mentioned that in the direct computation of the two-point function using the above vertex operator, certain $\sigma$-dependence remains, which was apparently subtracted in [26] as a part of the renormalization process. As was already remarked, the proper renormalization procedure for interacting theory is non-trivial and would require further careful understanding.

The analyses above indicate that, although the vertex operator (4.8) has the correct transformation property with respect to the target symmetry, it does not appear to have the correct worldsheet property even within the saddle point approximation.

Therefore, it is fair to say that for the LSGKP strings construction of the precise semi-classical vertex operators remains as an important future problem. In the next subsection, however, we will give a general discussion of how such vertex operator should be systematically constructed from the solution of the Hamilton-Jacobi equation. 


\subsection{Semi-classical vertex operator from WKB wave function}

Let us begin with the general relation between the vertex operator and the wave function. It is given by the so-called "state-operator correspondence", which is expressed by

$$
\left.\int \mathcal{D} X\right|_{X=X_{0}(\sigma) \text { at } \tau=\tau_{0}} V[X(z=0)] \exp \left(-\left.S\right|_{\tau<\tau_{0}}\right)=\Psi\left[X_{0}(\sigma)\right]
$$

Here and hereafter, we use the worldsheet coordinate $z=e^{\tau+i \sigma}$. On the left hand side, $V[X(z=0)]$ is the vertex operator inserted at $z=0, \exp \left(-\left.S\right|_{\tau<\tau_{0}}\right)$ is the contribution of the action within a disk of radius $e^{\tau_{0}}$ around $z=0$, and the path integral is performed over the string configuration with the prescribed behavior on the circumference at $\tau=\tau_{0}$ of the disk. This quantity equals the right hand side $\Psi\left[X_{0}(\sigma)\right]$, which represents the wave function for the degrees of freedom living on the circumference.

In the cases where the saddle point approximation is adequate, the path integral can be replaced by the value at the saddle and the formula simplifies to

$$
\ln V\left[X_{c l}(z=0)\right]-\left.S_{c l}\right|_{\tau<\tau_{0}}=\ln \Psi\left[X_{c l}\left(\tau=\tau_{0}, \sigma\right)\right] .
$$

At the same time, the wave function can be expressed in terms of an appropriate solution $\mathcal{W}$ of the Hamilton-Jacobi equation as

$$
\ln \Psi\left[X\left(\tau=\tau_{0}, \sigma\right)\right]=-\mathcal{W}\left[X\left(\tau=\tau_{0}, \sigma\right)\right] .
$$

However, except for simple cases such as those in flat spacetime, there are difficulties in constructing the vertex operator using the relation (4.10) directly. First, non-linearity of the equations of motion for a string in curved spacetime makes it a highly non-trivial problem to obtain the appropriate solution of the Hamilton-Jacobi equation and express it in terms of the set of target space variables $X[\sigma]$. Second, even if one succeeds in obtaining the wave function, the contribution of the action $\left.S_{c l}\right|_{\tau<\tau_{0}}$ is in general a complicated function of $\tau_{0}$ and hence separating the vertex operator part properly is not an easy process.

These difficulties may be greatly alleviated if one can find a judicious canonical transformation of variables. Suppose we make such a transformation from $X$ to a new set of variables $\phi_{i}$. Then, the relation (4.10) becomes

$$
\ln V\left[\phi_{c l}(z=0)\right]-\left.\tilde{S}_{\phi, c l}\right|_{\tau<\tau_{0}}=\ln \Psi\left[\phi_{i, c l}\left(\tau=\tau_{0}\right)\right] .
$$

Here $\tilde{S}_{\phi_{i}}$ is the action for $\phi_{i}$, given by

$$
\tilde{S}_{\phi}=\int d \tau\left(\sum_{i} P_{\phi_{i}} \partial_{\tau} \phi_{i}-H\right)
$$


where $P_{\phi_{i}}$ are the momenta conjugate to $\phi_{i}$ and $H$ denotes the worldsheet energy. In particular, if we can choose $\left(P_{\phi_{i}}, \phi_{i}\right)$ to be action-angle variables, then $P_{\phi_{i}} \partial_{\tau} \phi_{i}$ becomes a number and the evaluation of the action part simplifies enormously. At the same time, the Hamilton-Jacobi equations reduce to

$$
\frac{\partial \mathcal{W}}{\partial \phi_{i}}=P_{\phi_{i}}=\text { constant }
$$

and hence the wave function can be constructed simply as

$$
\Psi\left[\phi_{i}\right]=\exp \left[-\sum_{i} P_{\phi_{i}} \phi_{i}\right] .
$$

Note that in this description, even for a system like a string which has infinite degrees of freedom, it is possible that for certain configurations only a finite number of $P_{\phi_{i}}$ are non-vanishing and hence effectively we only need to deal with a system with finite degrees of freedom.

In making use of such canonical transformations, it is important to keep in mind that $V\left[X_{c l}(\sigma)\right]$ and $V\left[\phi_{i, c l}\right]$ are not in general equal. Indeed while $V[X(\sigma)]$ is expressed as

$$
V[X(\sigma)]=\langle X(\sigma)|V| 0\rangle
$$

where $|0\rangle$ denotes the $S L(2, R)$ invariant vacuum, the quantity $V\left[\phi_{i}\right]$ is given by

$$
V\left[\phi_{i}\right]=\left\langle\phi_{i}|V| 0\right\rangle=\int \mathcal{D} X\left\langle\phi_{i} \mid X\right\rangle\langle X|V| 0\rangle,
$$

which is obviously different from (4.16). In cases where we may use the saddle point approximation, $V\left[\phi_{i}\right]$ reduces to $V\left[\phi_{i}\right]=\left\langle\phi_{i} \mid X\right\rangle\langle X|V| 0\rangle$, where $X$ is the saddle point configuration. Even in such a situation the factor $\left\langle\phi_{i} \mid X\right\rangle$ may produce a finite difference ${ }^{13}$.

Although we have not yet been able to construct the proper vertex operator for the LSGKP string through above procedure, it is quite instructive to demonstrate the validity of the argument by using a specific solvable example. It is a rotating folded string solution in flat space [45] given by

$$
\begin{aligned}
& X^{\mu}=-i k^{\mu} \ln |z|, \quad \mu \neq 1,2, \\
& X \equiv X_{1}+i X_{2}=\frac{\omega}{2 i}(z-\bar{z}), \quad \bar{X} \equiv X_{1}-i X_{2}=\frac{\omega}{2 i}(1 / z-1 / \bar{z}) .
\end{aligned}
$$

(For simplicity we have set $\alpha^{\prime}=1$.) We will treat the zero mode and the non-zero mode separately, as they are completely decoupled in this case.

\footnotetext{
${ }^{13}$ In the case of a string in flat spacetime this factor turns out to be unity.
} 
For the zero mode, we can identify the angle variable as $x^{\mu}=\frac{1}{2 \pi} \int d \sigma X^{\mu}$ and the solution of the Hamilton-Jacobi equation is given by

$$
\mathcal{W}_{\text {zero mode }}=-i k_{\mu} x^{\mu}+\frac{k^{2}}{2} \tau
$$

Then the relation (4.10) for the zero mode part reads

$$
\log V_{\text {zero mode }}+\frac{k^{2}}{2}\left(\tau_{0}-\log \epsilon\right)=i k_{\mu} x^{\mu}\left(\tau_{0}\right)-\frac{k^{2}}{2} \tau_{0} .
$$

Note that to regularize the divergence, we have cut off the integration region for the action by $\tau=\log \epsilon$. Now if we use the relation $x^{\mu}\left(\tau=\tau_{0}\right)+i k^{\mu}\left(\tau_{0}-\log \epsilon\right)=i k_{\mu} x^{\mu}(\tau=\log \epsilon)=$ $i k_{\mu} X^{\mu}(\tau=\log \epsilon)+\mathcal{O}(\epsilon)$, the vertex operator can be written as

$$
\log V_{\text {zero mode }}=i k_{\mu} X^{\mu}(\tau=\log \epsilon)-\frac{k^{2}}{2} \log \epsilon .
$$

This vertex, in addition to the usual expression $i k_{\mu} X^{\mu}$, contains a divergent term of the form $-\frac{k^{2}}{2} \log \epsilon$. But this is the correct form because this precisely cancels the divergence produced by cutting off the area at $\tau=\epsilon$. Therefore, as we have pointed out already, the total amplitude is finite.

Let us now discuss the non-zero mode part. From the mode expansion

$$
\begin{array}{r}
X^{\mu}=x^{\mu}+i \sum_{n \neq 0} \frac{1}{n}\left(\alpha_{n}^{\mu} e^{-i n \sigma}+\tilde{\alpha}_{n}^{\mu} e^{i n \sigma}\right) \\
\partial_{\tau} X^{\mu}=-i p^{\mu}-\frac{i}{2} \sum_{n \neq 0}\left(\alpha_{n}^{\mu} e^{-i n \sigma}-\tilde{\alpha}_{n}^{\mu} e^{i n \sigma}\right)
\end{array}
$$

we can identify the relevant angle variables $\theta, \tilde{\theta}$ and the corresponding action variables $J, \tilde{J}$ as

$$
\begin{aligned}
\theta & \equiv \log \left(\alpha_{-1}^{1}+i \alpha_{-1}^{2}\right), & J & \equiv-\frac{1}{2}\left(\alpha_{-1}^{1}+i \alpha_{-1}^{2}\right)\left(\alpha_{-1}^{1}-i \alpha_{-1}^{2}\right), \\
\tilde{\theta} & \equiv \log \left(\tilde{\alpha}_{-1}^{1}+i \tilde{\alpha}_{-1}^{2}\right), & \tilde{J} & \equiv-\frac{1}{2}\left(\tilde{\alpha}_{-1}^{1}+i \tilde{\alpha}_{-1}^{2}\right)\left(\tilde{\alpha}_{-1}^{1}-i \tilde{\alpha}_{-1}^{2}\right) .
\end{aligned}
$$

For the rotating string solution above, action variables for all the other modes can be set to zero. The solution of the Hamilton-Jacobi equation is given by

$$
\mathcal{W}_{\text {oscillator }}=-\omega^{2} \tau-\frac{\omega^{2}}{2} \theta-\frac{\omega^{2}}{2} \tilde{\theta}
$$

In this case there is no contribution from the action $\tilde{S}$ and hence the vertex operator for this part is obtained through (4.10) as

$$
\begin{aligned}
\log V_{\text {oscillator }} & =\omega^{2} \tau+\frac{\omega^{2}}{2} \theta+\frac{\omega^{2}}{2} \tilde{\theta} \\
& =\frac{\omega^{2}}{2} \log \left(\int \frac{d z}{2 \pi i z} \partial X \int \frac{d \bar{z}}{2 \pi i \bar{z}} \bar{\partial} X\right) .
\end{aligned}
$$


Since the spin of this string is given by $S=\omega^{2}$, we finally obtain the expression

$$
V_{\text {oscillator }}=(\partial X \bar{\partial} X)^{S / 2},
$$

which is quite reasonable.

As it should be clear from the above demonstration, in order to construct the correct vertex operator, one must properly take into account the contribution of the action in the application of the state-operator correspondence. This applies to the GKP string as well: One should find appropriate action-angle variables and then apply the formula (4.10), which includes the effect of the action.

\section{$5 \quad$ Summary and future directions}

In this work, we have discussed how to evaluate the three point functions of heavy string states, carrying large quantum numbers of order $\sqrt{\lambda}$, by the use of the saddle point method. By adapting the powerful integrability-based method invented previously for the gluon scattering problem and making several non-trivial modifications, we have developed a way to compute the regularized area, which is the essential part of the $S L(2, C)$ invariant three point coupling, without the knowledge of the explicit form of the saddle point configuration. In particular, we have applied the method to the case of large spin limit of the GKP strings and were able to express the regularized area explicitly in terms of the parameters $\kappa_{i}$ that characterize the external GKP states. Reliable computation of the remaining $S L(2, C)$ variant part of the three point function, which contains the contributions of the vertex operators, must await future investigations, since at present time the precise form of the semi-classical vertex operator for the GKP string is not known. Nevertheless, we have presented a general discussion of how such vertex operators should be constructed systematically using the semi-classical wave functions obeying the Hamilton-Jacobi equations and gave an explicit example to illustrate the method.

There are some possible directions for future work.

One important task, of course, is to analyze the Hamilton-Jacobi equation relevant to the LSGKP string by developing a judicious integrability-based method and find the precise form of the semi-classical vertex operator for LSGKP. This will allow us to complete the computation of the three point functions of these states, which we set out to perform in this work. Also it would be desirable to extend the computation to the case of general GKP strings, without taking the large spin limit. Such investigations are now in progress.

Another obvious extension of our work is the computation of higher point functions, in particular that of four point functions of LSGKP strings. As already remarked in the 
main text, the generalized Riemann bilinear identity that we derived in this work should be indispensable for that purpose. It would be quite interesting if one can understand such characteristic property of the conformal field theory as the crossing symmetry etc. from the string theory side at strong 't Hooft coupling.

Clearly, the method that we developed in this article should be applicable to various other types of heavy strings, which for example belong to the class of so-called finite gap solutions. Successful applications in that direction would deepen our understanding of the integrability structure of string theory in curved spacetime.

Such study of the finite gap solutions might also lead to the discovery of some exact three-pronged string solution, which will undoubtedly be useful in performing and checking the calculation of three point functions.

Finally, it should be important to study how one can compute the next order in the $1 / \sqrt{\lambda}$ expansion, making use of the integrability structure of the system.

We hope to return to discuss progress in some of these problems in near future.

\section{Acknowledgment}

We are grateful to R. Janik for useful comments on the manuscript and E. Buchbinder and A. Tseytlin for raising clarifying questions. The research of Y.K. is supported in part by the Grant-in-Aid for Scientific Research (B) No. 20340048, while that of S.K. is supported in part by JSPS Research Fellowship for Young Scientists, from the Japan Ministry of Education, Culture, Sports, Science and Technology.

\section{Appendix A: Regularized area for general configura- tion of $\kappa_{i}$ 's}

Below we give some details of the analysis needed for the computation of the regularized area for more general configuration of the parameters $\kappa_{i}$ than discussed in the main text, namely the cases where the triangle inequality is not satisfied. Specifically we consider, without loss of generality, the case where the parameters satisfy

$$
\kappa_{2}>\kappa_{1}+\kappa_{3}
$$

Results for other cases are obtained by permutations of $\kappa_{i}$ 's.

Compared to the case where the triangle inequality is satisfied, there are substantial changes in the configuration of the WKB curves and the position of the branch cuts as exhibited in figure A.1. The most crucial difference is that two poles, $z_{1}$ and $z_{3}$ 


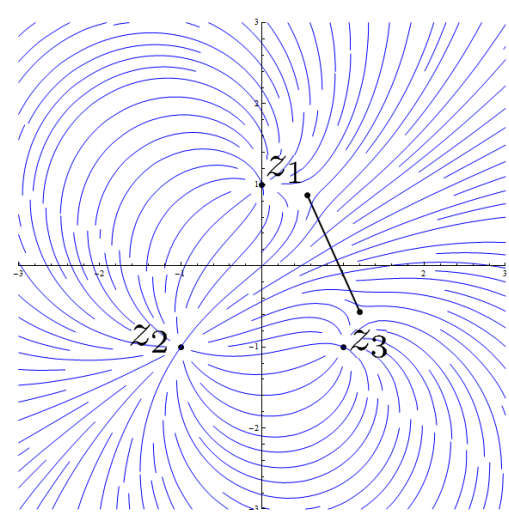

(a)

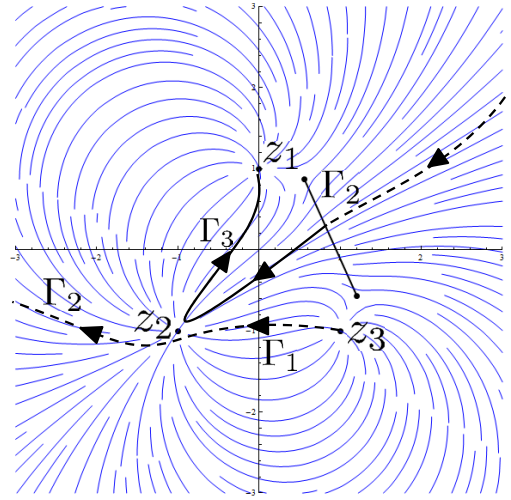

(b)

Figure A.1: (a) Behavior of WKB curves for the parameter regime where the triangle inequality is not satisfied. The parameters used are $\kappa_{1}=\kappa_{3}=1, \kappa_{2}=\sqrt{6}$ and $\operatorname{Im} \xi>0$. (b) Deformation of the contour connecting $\widehat{z}_{3}$ to $z_{1}$. The deformed contour consists of three parts $\Gamma_{1}, \Gamma_{2}$ and $\Gamma_{3}$.

in figure A.1, are no longer connected directly by a single WKB curve. Since reliable expansion must be made along WKB curves, we must deform the path connecting $z_{3}$ and $z_{1}$ appropriately as in figure A.1 so that the resultant path consists of the following three WKB curves $\Gamma_{1} \sim \Gamma_{3}$ :

$$
\begin{array}{ll}
\Gamma_{1}: & \text { connecting } \widehat{z}_{3} \text { and } \widehat{z}_{2} \\
\Gamma_{2}: & \text { connecting } \widehat{z}_{2} \text { and } z_{2} \\
\Gamma_{3}: & \text { connecting } z_{2} \text { and } z_{1}
\end{array}
$$

Contributions form $\Gamma_{1}$ and $\Gamma_{2}$, which lie respectively in the second and the first sheet, are easy to compute since we can show that, for $\operatorname{Im} \xi>0$, they are related to $\left\langle s_{2}, s_{3}\right\rangle^{-1}$ and $\left\langle s_{1}, s_{2}\right\rangle^{-1}$ respectively. On the other hand, evaluation of the contribution from $\Gamma_{2}$, which connects the points on top of each other on different sheets, requires care. Because $\widehat{z}_{2}$ is on the second sheet, the relevant quantity is

$$
\left\langle s_{2}, s_{2}^{\prime}\right\rangle
$$

where $s_{2}^{\prime}$ is the solution which can be obtained from $s_{2}$ by the analytic continuation along a path encircling $z_{1}$ clockwise. Now since $s_{2}$ is related to the plus-minus solutions in the following way,

$$
\begin{aligned}
& \operatorname{Im} \xi>0 \Rightarrow s_{2} \sim 2_{-}, \\
& \operatorname{Im} \xi<0 \Rightarrow s_{2} \sim 2_{+},
\end{aligned}
$$

we need to evaluate $\left\langle 2_{-}, 2_{-}^{\prime}\right\rangle$ and $\left\langle 2_{+}, 2_{+}^{\prime}\right\rangle$, where $2_{ \pm}^{\prime}$ are obtained from $2_{ \pm}$by the same 
analytic continuation as for $s_{2}^{\prime}$. Since $2_{-}$can be expressed in terms of the solutions $1_{ \pm}$as

$$
2_{-}=-\left\langle 1_{-}, 2_{-}\right\rangle 1_{+}+\left\langle 1_{+}, 2_{-}\right\rangle 1_{-},
$$

$2_{-}^{\prime}$ can be calculated as

$$
2_{-}^{\prime}=-\left\langle 1_{-}, 2_{-}\right\rangle e^{-i \rho_{1}(\xi)} 1_{+}+\left\langle 1_{+}, 2_{-}\right\rangle e^{+i \rho(\xi)} 1_{-} .
$$

Therefore, the product $\left\langle 2_{-}, 2_{-}^{\prime}\right\rangle$ can be evaluated as

$$
\begin{aligned}
\left\langle 2_{-}, 2_{-}^{\prime}\right\rangle & =-2 i \sin \rho_{1}(\xi)\left\langle 1_{+}, 2_{-}\right\rangle\left\langle 1_{-}, 2_{-}\right\rangle \\
& =-\left(c A_{2}^{2} \sin ^{2}\left(-\rho_{2}\right)\right)^{-1}\left(\sin \left(\frac{-\rho_{1}+\left(-\rho_{2}\right)-\rho_{3}}{2}\right) \sin \left(\frac{-\rho_{1}+\left(-\rho_{2}\right)+\rho_{3}}{2}\right)\right) .
\end{aligned}
$$

Similarly, $\left\langle 2_{+}, 2_{+}^{\prime}\right\rangle$ can be evaluated as

$$
\left\langle 2_{+}, 2_{+}^{\prime}\right\rangle=4 c A_{2}^{2}\left(\sin \left(\frac{\rho_{1}+\left(-\rho_{2}\right)+\rho_{3}}{2}\right) \sin \left(\frac{\rho_{1}+\left(-\rho_{2}\right)-\rho_{3}}{2}\right)\right) .
$$

Combining, we obtain the following normalization independent quantity:

$$
\begin{aligned}
\left\langle 2_{+}, 2_{+}^{\prime}\right\rangle\left\langle 2_{-}, 2_{-}^{\prime}\right\rangle & =-4\left(\sin ^{2}\left(-\rho_{2}\right)\right)^{-1} \times \\
& {\left[\sin \left(\frac{-\rho_{1}+\left(-\rho_{2}\right)-\rho_{3}}{2}\right) \sin \left(\frac{\rho_{1}+\left(-\rho_{2}\right)+\rho_{3}}{2}\right)\right.} \\
& \left.\sin \left(\frac{-\rho_{1}+\left(-\rho_{2}\right)+\rho_{3}}{2}\right) \sin \left(\frac{\rho_{1}+\left(-\rho_{2}\right)-\rho_{3}}{2}\right)\right] .
\end{aligned}
$$

From this result, one can determine the relevant part of $\left\langle s_{2}, s_{2}^{\prime}\right\rangle$ as follows:

$$
\begin{aligned}
& \operatorname{Im} \xi>0 \\
& \qquad \begin{aligned}
\log \left\langle s_{2}, s_{2}^{\prime}\right\rangle_{n . s .} & =2 h\left(-\rho_{2}\right)-h\left(\frac{\rho_{1}+\left(-\rho_{2}\right)+\rho_{3}}{2}\right)-h\left(\frac{-\rho_{1}+\left(-\rho_{2}\right)-\rho_{3}}{2}\right) \\
& -h\left(\frac{-\rho_{1}+\left(-\rho_{2}\right)+\rho_{3}}{2}\right)-h\left(\frac{\rho_{1}+\left(-\rho_{2}\right)-\rho_{3}}{2}\right)
\end{aligned}
\end{aligned}
$$

$\operatorname{Im} \xi<0$

$$
\begin{aligned}
\log \left\langle s_{2}, s_{2}^{\prime}\right\rangle_{n . s .} & =-2 h\left(-\rho_{2}\right)+h\left(\frac{\rho_{1}+\left(-\rho_{2}\right)+\rho_{3}}{2}\right)+h\left(\frac{-\rho_{1}+\left(-\rho_{2}\right)-\rho_{3}}{2}\right) \\
& +h\left(\frac{-\rho_{1}+\left(-\rho_{2}\right)+\rho_{3}}{2}\right)+h\left(\frac{\rho_{1}+\left(-\rho_{2}\right)-\rho_{3}}{2}\right) .
\end{aligned}
$$

Finally, summing up the contributions from all three curves $\Gamma_{1} \sim \Gamma_{3}$, we find that the net result is simply given by making the following substitution to the expression for the $\kappa_{2}<\kappa_{1}+\kappa_{3}$ case:

$$
K\left(\frac{\kappa_{1}-\kappa_{2}+\kappa_{3}}{2}\right) \longmapsto-K\left(\frac{-\kappa_{1}+\kappa_{2}-\kappa_{3}}{2}\right) .
$$

From this result and the corresponding ones with $\kappa_{i}$ permuted, one can easily deduce the general formula given in (3.142). 


\section{References}

[1] J.M. Maldacena, "The large-N limit of superconformal field theories and supergravity", Adv. Theor. Math. Phys. 2 (1998) 231 [arXiv:hep-th/9711200].

[2] S.S. Gubser, I.R. Klebanov and A.M. Polyakov, "Gauge theory correlators from noncritical string theory", Phys. Lett. B 428 (1998) 105 [arXiv:hep-th/9802109].

[3] E. Witten, "Anti-de Sitter space and holography", Adv. Theor. Math. Phys. 2 (1998) 253 [arXiv:hep-th/9802150].

[4] S. Kovacs, "N=4 supersymmetric Yang-Mills theory and the AdS / SCFT correspondence," [arXiv:hep-th/9908171].

[5] G. Arutyunov and S. Frolov, "String hypothesis for the $A d S_{5} \times S^{5}$ mirror", JHEP 03 (2009) 152 [arXiv:0901.1417].

G. Arutyunov and S. Frolov, "Thermodynamic Bethe Ansatz for the $A d S_{5} \times S^{5}$ Mirror Model", JHEP 05 (2009) 068 [arXiv:0903.0141].

[6] N. Gromov, V. Kazakov and P. Vieira, "Exact Spectrum of Anomalous Dimensions of Planar $N=4$ Supersymmetric Yang-Mills Theory", Phys. Rev. Lett. 103 (2009) 131601 [arXiv:0901.3753].

N. Gromov, V. Kazakov, A. Kozak and P. Vieira, "Exact Spectrum of Anomalous Dimensions of Planar N = 4 Supersymmetric Yang-Mills Theory: TBA and excited states", Lett. Math. Phys. 91 (2010) 265 [arXiv:0902.4458].

[7] D. Bombardelli, D. Fioravanti and R. Tateo, "Thermodynamic Bethe Ansatz for planar AdS/CFT: a proposal", J. Phys. A 42 (2009) 375401 [arXiv:0902.3930].

[8] N. Beisertet al., "Review of AdS/CFT Integrability: An Overview," [arXiv: 1012.3982].

[9] I. Bena, J. Polchinski and R. Roiban, "Hidden symmetries of the AdS(5) x S**5 superstring," Phys. Rev. D 69, 046002 (2004) [arXiv:hep-th/0305116].

[10] T. McLoughlin, "Review of AdS/CFT Integrability, Chapter II.2: Quantum Strings in AdS5xS5," [arXiv:1012.3987].

[11] S.S. Gubser, I.R. Klebanov and A.M. Polyakov, "A semi-classical limit of the gauge/string correspondence", Nucl. Phys. B 636 (2002) 99 [arXiv:hep-th/0204051].

[12] L. F. Alday and J. Maldacena, "Minimal surfaces in AdS and the eight-gluon scattering amplitude at strong coupling," [arXiv:0903.4707].

[13] L. F. Alday and J. Maldacena, "Null polygonal Wilson loops and minimal surfaces in Anti-de-Sitter space," JHEP 0911, 082 (2009) [arXiv:0904.0663].

[14] D. Gaiotto, G. W. Moore and A. Neitzke, "Wall-crossing, Hitchin Systems, and the WKB Approximation", [arXiv:0907.3987].

[15] L. F. Alday, D. Gaiotto and J. Maldacena, "Thermodynamic Bubble Ansatz," JHEP 
1109, 032 (2011) [arXiv:0911.4708].

[16] L.F. Alday, J. Maldacena, A. Sever and P. Vieira, "Y-system for Scattering Amplitudes", J.Phys. A 43 (2010) 485401 [arXiv:1002.2459].

[17] S. Lee, S. Minwalla, M. Rangamani and N. Seiberg, "Three point functions of chiral operators in D =4, N=4 SYM at large N," Adv. Theor. Math. Phys. 2, 697 (1998) [arXiv:hep-th/9806074].

[18] K. Okuyama and L. S. Tseng, "Three-point functions in N $=4$ SYM theory at one-loop," JHEP 0408, 055 (2004) [arXiv: hep-th/0404190].

[19] R. Roiban and A. Volovich, "Yang-Mills correlation functions from integrable spin chains," JHEP 0409, 032 (2004) [arXiv: hep-th/0407140].

[20] L. F. Alday, J. R. David, E. Gava and K. S. Narain, "Structure constants of planar N = 4 Yang Mills at one loop," JHEP 0509, 070 (2005) [arXiv:hep-th/0502186].

[21] J. Escobedo, N. Gromov, A. Sever and P. Vieira, "Tailoring Three-Point Functions and Integrability," JHEP 1109, 028 (2011) [arXiv: 1012.2475].

J. Escobedo, N. Gromov, A. Sever and P. Vieira, "Tailoring Three-Point Functions and Integrability II. Weak/strong coupling match," JHEP 1109, 029 (2011) [arXiv: 1104.5501].

[22] G. Georgiou, "SL(2) sector: weak/strong coupling agreement of three-point correlators," JHEP 1109, 132 (2011). [arXiv: 1107.1850].

[23] J. Caetano, J. Escobedo, "On four-point functions and integrability in N=4 SYM: from weak to strong coupling," JHEP 1109, 080 (2011). [arXiv:1107.5580].

[24] A. Tsuji, "Holography of Wilson loop correlator and spinning strings," Prog. Theor. Phys. 117, 557 (2007) [arXiv:hep-th/0606030].

[25] R. Janik, P. Surowka and A. Wereszczynski, "On correlation functions of operators dual to classical spinning string states", JHEP 05 (2010) 030 [arXiv:1002.4613].

[26] E.I. Buchbinder and A.A. Tseytlin, "On semiclassical approximation for correlators of closed string vertex operators in AdS/CFT", JHEP 08 (2010) 057 [arXiv: 1005. 4516].

[27] N. Dorey, "A Spin Chain from String Theory," Acta Phys. Polon. B39, 3081-3116 (2008). [arXiv:0805.4387].

N. Dorey, M. Losi, "Spiky Strings and Spin Chains," [arXiv:0812.1704].

N. Dorey, M. Losi, "Spiky Strings and Giant Holes," JHEP 1012, 014 (2010). [arXiv:1008.5096].

[28] K. Zarembo, "Holographic three-point functions of semiclassical states", JHEP 09 (2010) 030 [arXiv: 1008.1059].

[29] M.S. Costa, R. Monteiro, J.E. Santos and D. Zoakos, "On three-point correlation functions in the gauge/gravity duality", JHEP 11 (2010) 141 [arXiv:1008.1070]. 
[30] R. Roiban and A.A. Tseytlin, "On semiclassical computation of 3-point functions of closed string vertex operators in $\mathrm{AdS}_{5} \times \mathrm{S}^{5}$ ", Phys.Rev.D 82 (2010) 106011 [arXiv: 1008.4921].

[31] R. Hernandez, "Three-point correlation functions from semiclassical circular strings", J.Phys. A 44 (2011) 085403 [arXiv:1011.0408].

[32] S. Ryang, "Correlators of Vertex Operators for Circular Strings with Winding Numbers in $A d S_{5} \times S^{5} "$, JHEP 01 (2011) 092 [arXiv: 1011.3573].

[33] G. Georgiou, "Two and three-point correlators of operators dual to folded string solutions at strong coupling", JHEP 02 (2011) 046 [arXiv:1011.5181].

[34] J.G. Russo and A.A. Tseytlin, "Large spin expansion of semiclassical 3-point correlators in $A d S_{5} \times S^{5}$ ", JHEP 02 (2011) 029 [arXiv: 1012.2760].

[35] C. Park and B.-H. Lee, "Correlation functions of magnon and spike", Phys. Rev. D 83 (2011) 126004 [arXiv:1012.3293].

[36] E.I. Buchbinder and A.A. Tseytlin, "Semiclassical four-point functions in $A d S_{5} \times$ $S^{5 ",}$ JHEP 02 (2011) 072 [arXiv: 1012.3740].

[37] D. Bak, B. Chen, J.-B. Wu, "Holographic Correlation Functions for Open Strings and Branes", JHEP 06 (2011) 014 [arXiv: 1103.2024].

[38] A. Bissi, C. Kristjansen, D. Young and K. Zoubos, "Holographic three-point functions of giant gravitons", JHEP 06 (2011) 085 [arXiv: 1103.4079].

[39] D. Arnaudov, R.C. Rashkov and T. Vetso, "Three and four-point correlators of operators dual to folded string solutions in $A d S_{5} \times S^{5}$ ", Int. J. Mod. Phys. A 26 (2011) 3403 [arXiv:1103.6145].

[40] R. Hernandez, "Three-point correlators for giant magnons", JHEP 05 (2011) 123 [arXiv:1104.1160].

[41] C. Ahn and P. Bozhilov, "Three-point Correlation functions of Giant magnons with finite size", Phys. Lett. B 702 (2011) 286 [arXiv:1105.3084].

[42] T. Klose and T. McLoughlin, "A light-cone approach to three-point functions in $A d S_{5} \times S^{5 ",},[\operatorname{arXiv}: 1106.0495]$.

[43] K. Pohlmeyer, "Integrable Hamiltonian Systems and Interactions Through Quadratic Constraints," Commun. Math. Phys. 46, 207 (1976).

[44] R. A. Janik and A. Wereszczynski, "Correlation functions of three heavy operators - the AdS contribution," [arXiv:1109.6262].

[45] A. A. Tseytlin, "On semiclassical approximation and spinning string vertex operators in $A d S_{5} \times S^{5}$," Nucl. Phys. B664, 247-275 (2003). [arXiv:hep-th/0304139].

[46] Y. Kazama and S. Komatsu, "Erratum: On holographic three point functions for GKP strings from integrability", JHEP 06 (2012) 150 